\title{
LOCALIZATION OF SOLUTION OF THE PROBLEM OF TWO-DIMENSIONAL THEORY OF ELASTICITY WITH THE USE OF B-SPLINE DISCRETE-CONTINUAL FINITE ELEMENT METHOD
}

\author{
Marina L. Mozgaleva, Pavel A. Akimov, Taymuraz B. Kaytukov \\ National Research Moscow State University of Civil Engineering, Moscow, RUSSIA
}

\begin{abstract}
Localization of solution of the problem of two-dimensional theory of elasticity with the use of B-spline discrete-continual finite element method (specific version of wavelet-based discrete-continual finite element method) is under consideration in the distinctive paper. The original operational continual and discrete-continual formulations of the problem are given, some actual aspects of construction of normalized basis functions of a B-spline are considered, the corresponding local constructions for an arbitrary discrete-continual finite element are described, some information about the numerical implementation and an example of analysis are presented.
\end{abstract}

Keywords: localization, wavelet-based discrete-continual finite element method,

B-spline discrete-continual finite element method, discrete-continual finite element method,

finite element method, B-spline, numerical solution, two-dimensional theory of elasticity, structural analysis.

\section{ЛОКАЛИЗАЦИЯ РЕШЕНИЯ ДВУМЕРНОЙ ЗАДАЧИ ТЕОРИИ УПРУГОСТИ НА ОСНОВЕ ВЕЙВЛЕТ-РЕАЛИЗАЦИИ ДИСКРЕТНО-КОНТИНУАЛЬНОГО МЕТОДА КОНЕЧНЫХ ЭЛЕМЕНТОВ С ИСПОЛЬЗОВАНИЕМ В-СПЛАЙНОВ}

\author{
М.Л. Мозгалева, П.А. Акимов, Т.Б. Кайтуков \\ Национальный исследовательский Московский государственный строительный университет, \\ г. Москва, РОССИЯ
}

\begin{abstract}
Аннотация: В настоящей статье рассматривается локализация решения двумерной задачи теории упругости на основе вейвлет-реализации дискретно-континуального метода конечных элементов с использованием В-сплайнов. Приведены исходные операторные континуальная и дискретно-континуальная постановки задачи, рассмотрены некоторые актуальные вопросы построения нормализованных базисных функций В-сплайна, описаны соответствующие локальные построения для произвольного дискретно-континуального конечного элемента, представлены некоторые сведения о численной реализации и пример расчета.
\end{abstract}

Ключевые слова: локализация, вейвлет-реализация метода конечных элементов, дискретно-континуальный метод конечных элементов, метод конечных элементов, В-сплайны, численное решение, двумерная задача теории упругости, расчеты конструкций.

\section{INTRODUCTION}

As we have already mentioned $[1,2]$, the B-spline in a given simple knot sequence can be constructed by employing piecewise polynomials between the knots and joining them together at the knots [1-3].

For instance, compared with commonly used Daubechies wavelets [4-8] B-spline wavelet on interval (BSWI) has explicit expressions, facili- 
tating the calculation of coefficient integration and differentiation [1-3]. Besides, the multiresolution and localization properties of BSWI can also supply some superiority for engineering structural analysis [1-3]. The early applications of spline can be found in papers of $\mathrm{H}$. Antes [9], J.G. Han [10, 11, 27], Y. Huang [10, 11], W.X. Ren $[10,11]$. The spline wavelet finite element method was further developed in papers of D.P. Chen [28], X.F. Chen [12, 13, 15-18, 23, 24, 26], H.B. Dong [23], J.G. Han [25], Y.M. He [17], Z.H. He [18], Z.J. He [12, 13, 15-17, 23, 24, 26], Y. Huang [25, 27], Z.S. Jiang [22], B. Li [13, 15, 17, 23], M. Liang [19, 21], J.Q. Long [20], G. Ma [20], T. Matsumoto [20, 22], S.T. Mau [30], H.H. Miao [15], Q,M. Mo [18], T.H.H. Pian [28-30], K.Y. Qi [23], W.X. Ren [25, 27], K. Sumihara [29], P. Tong [30], Y.W. Wang [22], J.W. Xiang [12-14, 17-22], Z.B. Yang [15, 16, 24], X.W. Zhang [16, 24, 26], Y.H. Zhang [12], Y.T. Zhong [14].

As is known, generally the structural analysis normally require accurate computer-intensive calculations using numerical (discrete) methods. The field of application of discrete-continual finite element method (DCFFEM), proposed by A.B. Zolotov [33] and P.A. Akimov [31-33] comprises structures with regular (in particular, constant or piecewise constant) physical and geometrical parameters in some dimension (socalled "basic" direction (dimension)). Considering problems remain continual along "basic" direction while along other directions DCFEM presupposes finite element approximation. Solution of corresponding resultant multipoint boundary problems [34] for systems of ordinary differential equations with piecewise constant coefficients and immense number of unknowns is the most time-consuming stage of the computing, especially if we take into account the limitation in performance of personal computers, contemporary software and necessity to obtain correct semianalytical solution in a reasonable time.

High-accuracy solution at all points of the model is not required normally, it is necessary to find only the most accurate solution in some pre-known domains. Generally the choice of these domains is a priori data with respect to the structure being modelled. Designers usually choose domains with the so-called edge effect (with the risk of significant stresses that could potentially lead to the destruction of structures, etc.) and regions which are subject to specific operational requirements. It is obvious that the stress-strain state in such domains is of paramount importance. Specified factors along with the obvious needs of the designer or researcher to reduce computational costs by application of DCFEM cause considerable urgency of constructing of special algorithms for obtaining local solutions (in some domains known in advance) of boundary problems. Wavelet analysis provides effective and popular tool for such researches. Solution of the considering problem within multilevel wavelet analysis is represented as a composition of local and global components. Wavelet-based DCFEM is presented in papers of P.A. Akimov [35-42], M. Aslami [3840], T.B. Kaytukov, M.L. Mozgaleva [35-42] and O.A. Negrozov [38-40].

The distinctive paper is devoted to numerical solution of the problem of two-dimensional theory of elasticity with the use of B-spline DCFEM.

\section{FORMULATIONS OF THE PROBLEM}

In accordance with [1] let the constancy of the parameters of the problem be in the direction corresponding to $x_{2}$ (main direction). The operational formulation of the problem with the use of so-called method of extended domain [43], taking into account the selection of the main direction, is determined by the equation:

$$
L \bar{u}=\overline{\widetilde{F}}, \quad 0 \leq x_{1} \leq \ell_{1}, 0 \leq x_{2} \leq \ell_{2},
$$

where we have

$$
\begin{gathered}
L=-L_{v v} \partial_{2}^{2}+L_{u v} \partial_{2}+L_{u u} ; \\
L_{v v}=D_{2}^{T} C D_{2} ; \\
L_{u v}=\partial_{1}^{*} D_{1}^{T} C D_{2}-D_{2}^{T} C D_{1} \partial_{1} ; \\
L_{u u}=\partial_{1}^{*} D_{1}^{T} C D_{1} \partial_{1} ;
\end{gathered}
$$


Localization of Solution of the Problem of Two-Dimensional Theory of Elasticity with the Use of B-Spline DiscreteContinual Finite Element Method

$$
\begin{gathered}
\overline{\widetilde{F}}=\theta \bar{F}+\delta_{\Gamma} \bar{f} ; \\
\theta\left(x_{1}, x_{2}\right)=\left\{\begin{array}{cc}
1, & \left(x_{1}, x_{2}\right) \in \Omega \\
0, & \left(x_{1}, x_{2}\right) \notin \Omega ;
\end{array}\right. \\
\delta_{\Gamma}\left(x_{1}, x_{2}\right)=\partial \theta / \partial \bar{n} ;
\end{gathered}
$$

$\Omega$ is the domain, occupied by structure;

$\Omega=\left\{\left(x_{1}, x_{2}\right): 0<x_{1}<\ell_{1} ; 0<x_{2}<\ell_{2}\right\} ;$

$\ell_{1}, \ell_{2}$ are corresponding dimensions of extended domain (linear dimensions of considering structure $) ; \quad x=\left(x_{1}, x_{2}\right) ; x_{1}, x_{2}$ are Cartesian coordinates; $\theta\left(x_{1}, x_{2}\right)$ is characteristic function of domain $\Omega ; \delta_{\Gamma}=\delta_{\Gamma}\left(x_{1}, x_{2}\right)$ is the delta function of boundary $\Gamma=\partial \Omega ; \quad \bar{n}=\left[\begin{array}{ll}n_{1} & n_{2}\end{array}\right]^{T}$ is boundary normal vector; $\bar{u}$ is the vector of displacements (unknown vector function),

$$
\begin{aligned}
& \bar{u}=\left[\begin{array}{l}
u_{1} \\
u_{2}
\end{array}\right] ; \\
& D_{1}=\left[\begin{array}{ll}
1 & 0 \\
0 & 0 \\
0 & 1
\end{array}\right] ; \quad D_{2}=\left[\begin{array}{ll}
0 & 0 \\
0 & 1 \\
1 & 0
\end{array}\right] ; \\
& C=\left[\begin{array}{ccc}
2 \mu+\lambda & \lambda & 0 \\
\lambda & 2 \mu+\lambda & 0 \\
0 & 0 & \mu
\end{array}\right] \text {; }
\end{aligned}
$$

$\mu$ and $\lambda$ are Lame parameters; $\bar{F}$ is the load vector in domain $\Omega ; \bar{f}$ is the corresponding boundary load vector; $\partial_{s}=\partial / \partial x_{s}, s=1,2$; $\partial_{s}^{*}=-\partial / \partial x_{s}, s=1,2$.

Let us introduce the following notations

$$
\begin{gathered}
\bar{v}=\partial_{2} \bar{u}=\left[\begin{array}{l}
v_{1} \\
v_{2}
\end{array}\right] ; \\
\bar{u}^{\prime}=\partial_{2} \bar{u} ; \quad \bar{v}^{\prime}=\partial_{2} \bar{v} .
\end{gathered}
$$

Thus we can rewrite (1.1):

$$
L_{u u} \bar{u}+L_{u v} \bar{v}-L_{v v} \partial_{2} \bar{v}=\overline{\widetilde{F}} .
$$

Finally we obtain system of differential equations with operational coefficients:

$$
\left\{\begin{array}{l}
\bar{u}^{\prime}=\bar{v} \\
\bar{v}^{\prime}=L_{v v}^{-1} L_{u u} \bar{u}+L_{v v}^{-1} L_{u v} \bar{v}-L_{v v}^{-1} \overline{\widetilde{F}}
\end{array}\right.
$$

or

$$
\bar{z}^{\prime}=\widetilde{L} \bar{z}+\overline{\bar{F}}
$$

where

$$
\begin{gathered}
\widetilde{L}=\left[\begin{array}{cc}
0 & E \\
L_{v v}^{-1} L_{u u} & L_{v v}^{-1} L_{u v}
\end{array}\right] ; \quad \overline{\widehat{F}}=\left[\begin{array}{c}
0 \\
-L_{v v}^{-1} \overline{\widetilde{F}}
\end{array}\right] \\
\bar{z}=\left[\begin{array}{c}
\bar{u} \\
\bar{v}
\end{array}\right] ; \quad E=\left[\begin{array}{cc}
1 & 0 \\
0 & 1
\end{array}\right]
\end{gathered}
$$

The system of equations (1.17) is supplemented by boundary conditions, which are set in sections with coordinates $x_{2}^{1}=0$ and $x_{2}^{2}=\ell_{2}$.

\section{SOME ASPECTS OF THE \\ CONSTRUCTION OF NORMALIZED BASIS FUNCTIONS OF THE B-SPLINE}

The construction of B-spline basic functions is determined by the recursive Cox-de Boer formulas [1]:

$$
\begin{aligned}
& k=1: \quad \varphi_{i, 1}(t)=\left\{\begin{array}{l}
1, \quad x_{i} \leq t<x_{i+1}, \\
0, t<x_{i} \vee t \geq x_{i+1}
\end{array},\right. \\
& \begin{aligned}
\varphi_{i, k}(t)= & \frac{\left(t-x_{i}\right) \varphi_{i, k-1}(t)}{x_{i+k-1}-x_{i}}+ \\
& +\frac{\left(x_{i+k}-t\right) \varphi_{i+1, k-1}(t)}{x_{i+k}-x_{i+1}} .
\end{aligned}
\end{aligned}
$$

We will consider such a construction for the case $x_{i}=i$ are integers. Let us note that,

$$
\varphi_{i, k}(t)=\varphi_{0, k}(t-i)
$$

and therefore, recursive formulas (2.1)-(2.2) can be represented in the form 


$$
\begin{aligned}
& k=1: \quad \varphi_{0,1}(t)=\left\{\begin{array}{lr}
1, & 0 \leq t<1 \\
0, & t<0 \vee t \geq 1 ;
\end{array}\right. \\
& \begin{aligned}
k \geq 2: \quad \varphi_{0, k}(t)= & \frac{1}{k-1}\left[t \cdot \varphi_{0, k-1}(t)+\right. \\
& \left.+(k-t) \varphi_{0, k-1}(t-1)\right] .
\end{aligned}
\end{aligned}
$$

The function $\varphi_{0,1}(t)$ can be represented by formula

$$
\varphi_{0,1}(t)=\frac{1}{2}[\operatorname{sign}(t)-\operatorname{sign}(t-1)] .
$$

Let us denote by $\Delta_{1}$ the operator of the first difference. Then we have

$$
\varphi_{0,1}(t)=-\frac{1}{2} \Delta_{1} \operatorname{sign}(t)
$$

We can substitute formula (2.5) into (2.4) in order to determine $\varphi_{0,2}(t)$ :

$$
\begin{aligned}
& \varphi_{0,2}(t)=1 \cdot\left[t \cdot \varphi_{0,1}(t)+(2-t) \varphi_{0,1}(t-1)\right]= \\
& =\frac{1}{2}\{t \cdot[\operatorname{sign}(t)-\operatorname{sign}(t-1)]+ \\
& (2-t)[\operatorname{sign}(t-1)-\operatorname{sign}(t-2)]\}= \\
& =\frac{1}{2}[t \operatorname{sign}(t)-2(t-1) \operatorname{sign}(t-1)+ \\
& (t-2) \operatorname{sign}(t-2)]=\frac{1}{2}[|t|-2|t-1|+|t-2| \text {. }
\end{aligned}
$$

Let us denote by $\Delta_{2}$ the operator of the second difference. Then we have

$\varphi_{0,2}(t)=\frac{1}{2}\left[|t|-2|t-1|+|t-2|=\frac{1}{2} \Delta_{2}|t-1|\right.$.

We can define function $\varphi_{0,3}(t)$ :

$$
\varphi_{0,3}(t)=\frac{1}{2}\left[t \cdot \varphi_{0,2}(t)+(3-t) \varphi_{0,2}(t-1)\right] .
$$

Omitting intermediate calculations, we get

$$
\begin{aligned}
& \varphi_{0,3}(t)=\frac{1}{4} {[t \cdot|t|-3(t-1)|t-1|+} \\
&+3(t-2)|t-2|-(t-3)|t-3|]= \\
&=-\frac{1}{2 !} \frac{1}{2} \Delta_{1} \Delta_{2}((t-1)|t-1|) .
\end{aligned}
$$

Based on formulas (2.8) and (2.4), we can define the function

$$
\varphi_{0,4}(t)=\frac{1}{3}\left[t \cdot \varphi_{0,3}(t)+(4-t) \varphi_{0,3}(t-1)\right] .
$$

Omitting intermediate calculations, as a result we get

$$
\begin{aligned}
\varphi_{0,4}(t) & = \\
= & \frac{1}{2 \cdot 3} \cdot \frac{1}{2}\left[t^{2} \cdot|t|-4(t-1)^{2}|t-1|+\right. \\
+ & 6(t-2)^{2}|t-2|-4(t-3)^{2}|t-3|+ \\
+ & \left.(t-4)^{2}|t-4|\right]= \\
& =\frac{1}{3 !} \frac{1}{2}\left(\Delta_{2}\right)^{2}\left((t-2)^{2}|t-2|\right) .
\end{aligned}
$$

It can be proved that for even $k=2 m$ we have

$$
\varphi_{0, k}(t)=\frac{1}{(2 m-1) !} \frac{1}{2}\left(\Delta_{2}\right)^{m}\left((t-m)^{2 m-2}|t-m|\right)
$$

and for odd (uneven) $k=2 m+1$ we have

$\varphi_{0, k}(t)=-\frac{1}{(2 m) !} \frac{1}{2} \Delta_{1}\left(\Delta_{2}\right)^{m}\left((t-m)^{2 m-1}|t-1|\right)$.

Note that $\varphi_{0, k}(t)$ is a polynomial of degree $k-1$ with bounded support and, as follows from the difference operator, this support is equal to the interval $[0, k]$.

In addition, we should note the following property of B-spline basis functions:

$$
\sum_{i} \varphi_{0, k}(t-i) \equiv 1 \text { for arbitrary } t
$$




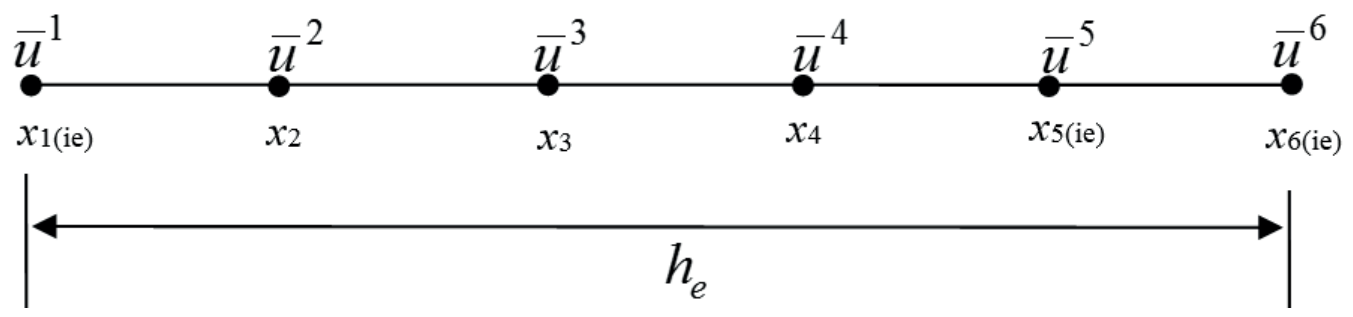

Figure 3.1. Finite element discretication for $N_{k}=5$ (sample).

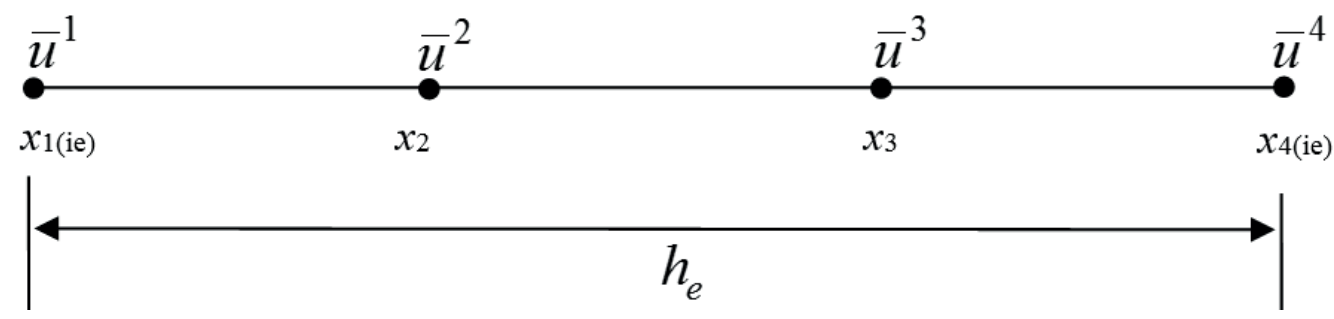

Figure 3.2. Finite element discretication for $N_{k}=3$ (sample).

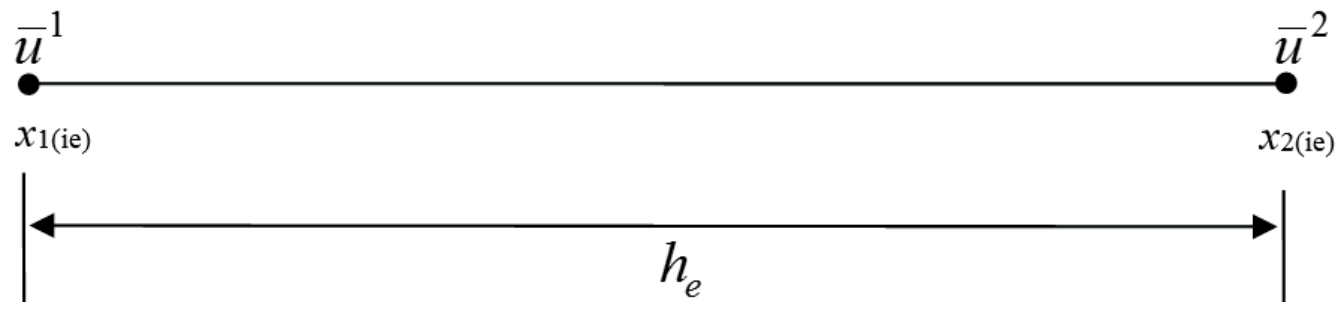

Figure 3.3. Finite element discretication for $N_{k}=1$ (sample).

\section{SOME GENERAL ASPECTS OF FINITE ELEMENT APPROXIMATION}

The discrete component of the numerical solution is represented by the direction along the axis corresponding to $x_{1}$. The fulfillment within an element (interval) for all components of a vector functions $\bar{u}$ and $\bar{v}$ (see $(1.10),(1.13))$ is the same. Therefore, let us use the following notation for simplicity:

$$
x=x_{1}, \ell=\ell_{1}, y=y(x),
$$

where $y=y(x)$ is unknown function (component of vector function).

Let us divide the interval $(0, \ell)$ segment into $N_{e}$ parts (elements). Therefore $h_{e}=\ell / N_{e}$ is the length of the element. Besides, let us also divide each element into $N_{k}$ parts. It should be noted that on the elements of the localization of the so- lution, parameter $N_{k}$ is of greater importance than on the other elements. For example, on 1ocalization elements, we can set $N_{k}=5$, i.e. unknown functions will be represented by polynomials (B-splines) of the 5th degree (Figure 3.1). Let us use the following notation system: $i_{e}$ is the element number; $N_{p}=N_{k}+1=6$ is the number of nodes within the element; $x_{1}\left(i_{e}\right)$ is the coordinate of the starting point of the $i_{e}$-th element; $x_{6}\left(i_{e}\right)$ is the coordinate of the end point of the $i_{e}$-th element. Thus, the number of unknowns per element with such approximation is equal to

$$
N_{i e}=2 N_{p}=12 \text {. }
$$

For the elements of localization we can take reduced number of $N_{k}$. For instance, if we take 
$N_{k}=3$ (Figure 3.2) we get $N_{p}=N_{k}+1=4$ and the number of unknowns per element with such approximation is equal to

$$
N_{i e}=2 N_{p}=8
$$

$x_{1}\left(i_{e}\right)$ is the coordinate of the starting point of the $i_{e}$-th element; $x_{4}\left(i_{e}\right)$ is the coordinate of the end point of the $i_{e}$-th element.

Besides, let us consider the case with $N_{k}=1$ (Figure 3.3). Therefor we have $N_{p}=N_{k}+1=2$ and the number of unknowns per element with such approximation is equal to

$$
N_{i e}=2 N_{p}=4,
$$

where $x_{1}\left(i_{e}\right)$ is the coordinate of the starting point of the $i_{e}$-th element; $x_{2}\left(i_{e}\right)$ is the coordinate of the end point of the $i_{e}$-th element.

\section{LOCAL CONSTRUCTIONS FOR ARBITRARY FINITE ELEMENT}

Let us introduce local coordinates:

$$
t=\left(x-x_{1(i e)}\right) / h_{e}, \quad x_{1(i e)} \leq x \leq x_{N_{p}(i e)}, 0 \leq t \leq 1 .
$$

In this case, we have the following relations:

$$
\begin{gathered}
x=x_{i} \Rightarrow t_{i}=\left(x_{i}-x_{1(i e)}\right) / h_{e}, \quad i=1, \ldots, N_{p} \\
\frac{d^{p}}{d x^{p}}=\frac{1}{h_{e}^{p}} \frac{d^{p}}{d t^{p}} ; \quad d x=h_{e} \cdot d t .
\end{gathered}
$$

Since the number of unknowns on the element is equal to $N_{i e}=12$, we use a B-spline of the fifth degree in order to represent the unknown deflection function.

Let us use the following notation:

$$
\varphi(t)=\varphi_{0,6}(t+3)
$$

$$
\begin{aligned}
\varphi(t) & =\frac{1}{5 !} \frac{1}{2}\left(\Delta_{2}\right)^{3}\left(t^{4}|t|\right)= \\
& =\frac{1}{5 ! \cdot 2}\left[(t+3)^{4}|t+3|-6(t+2)^{4}|t+2|+\right. \\
& +15(t+1)^{4}|t+1|-20 t^{4}|t|+ \\
& +15(t-1)^{4}|t-1|-6(t-2)^{4}|t-2|+ \\
& \left.+(t-3)^{4}|t-3|\right] .
\end{aligned}
$$

This function is a B-spline, symmetric with respect to $t=0$ and its support is defined by an interval $[-3,3]$ (Figure 4.1).

We take the following eight functions as basis functions on the unit interval (Figures 4.2, 4.3):

$$
\begin{gathered}
\varphi_{1}(t)=\varphi(t+2), \quad \varphi_{2}(t)=\varphi(t+1), \\
\varphi_{3}(t)=\varphi(t), \quad \varphi_{4}(t)=\varphi(t-1), \\
\varphi_{5}(t)=\varphi(t-2), \quad \varphi_{6}(t)=\varphi(t-3), \\
0 \leq t \leq 1 .
\end{gathered}
$$

Since the number of unknowns on the element is equal to $N_{i e}=8$, we use a B-spline of the third degree in order to represent the unknown deflection function.

Let us use the following notation:

$$
\begin{aligned}
& \varphi(t)=\varphi_{0,4}(t+4) ; \\
& \varphi(t)= \frac{1}{3 !} \frac{1}{2}\left(\Delta_{2}\right)^{2}\left(t^{2}|t|\right)= \\
&= \frac{1}{3 ! \cdot 2}\left[(t+2)^{2}|t+2|-4(t+1)^{2}|t+1|+\right. \\
&+6 t^{2}|t|-4(t-1)^{2}|t-1|+ \\
&\left.+(t-2)^{2}|t-2|\right] .
\end{aligned}
$$

This function is a B-spline, symmetric with respect to $t=0$ and its support is defined by an interval $[-2,2]$ (Figure 4.4).

We take the following four functions as basis functions on the unit interval (Figures 4.5):

$$
\begin{gathered}
\varphi_{1}(t)=\varphi(t+1), \quad \varphi_{2}(t)=\varphi(t), \\
\varphi_{3}(t)=\varphi(t-1), \quad \varphi_{4}(t)=\varphi(t-2), \\
0 \leq t \leq 1 .
\end{gathered}
$$


Localization of Solution of the Problem of Two-Dimensional Theory of Elasticity with the Use of B-Spline DiscreteContinual Finite Element Method

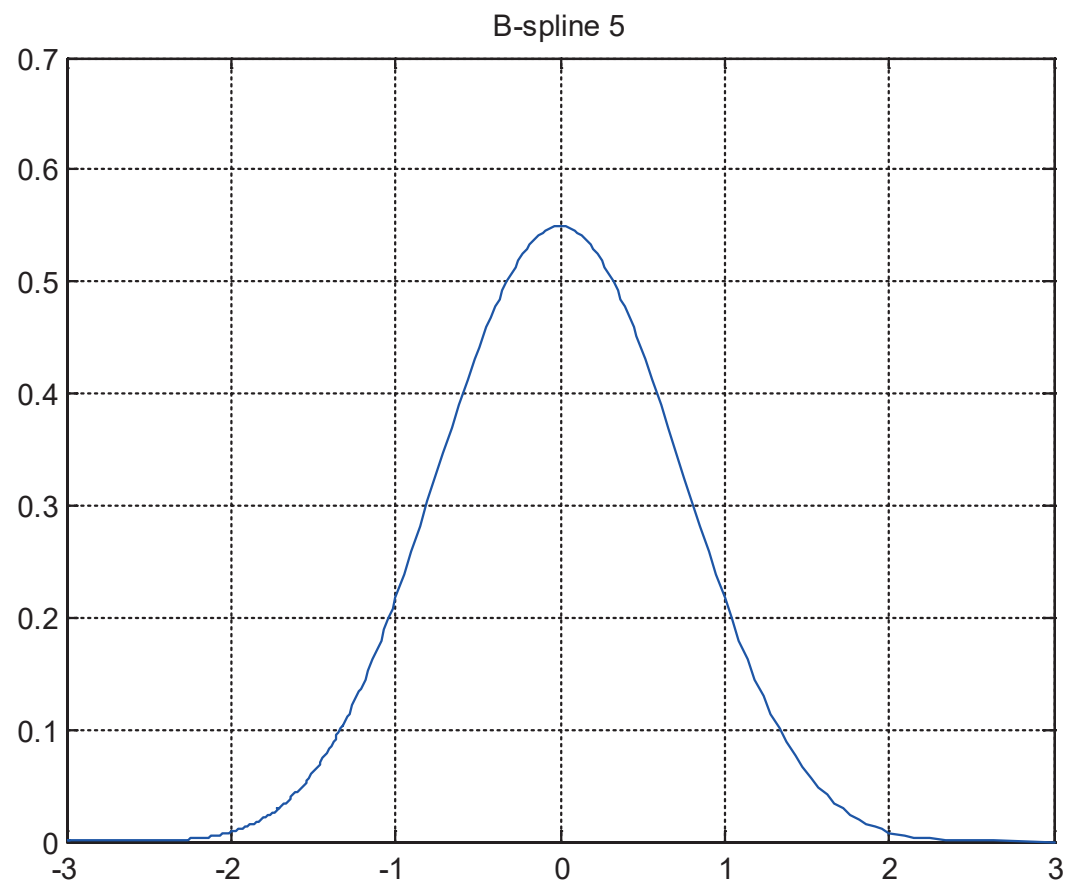

Figure 4.1. B-spline of the fifth order $\varphi(t)=\varphi_{0,6}(t+3)$.

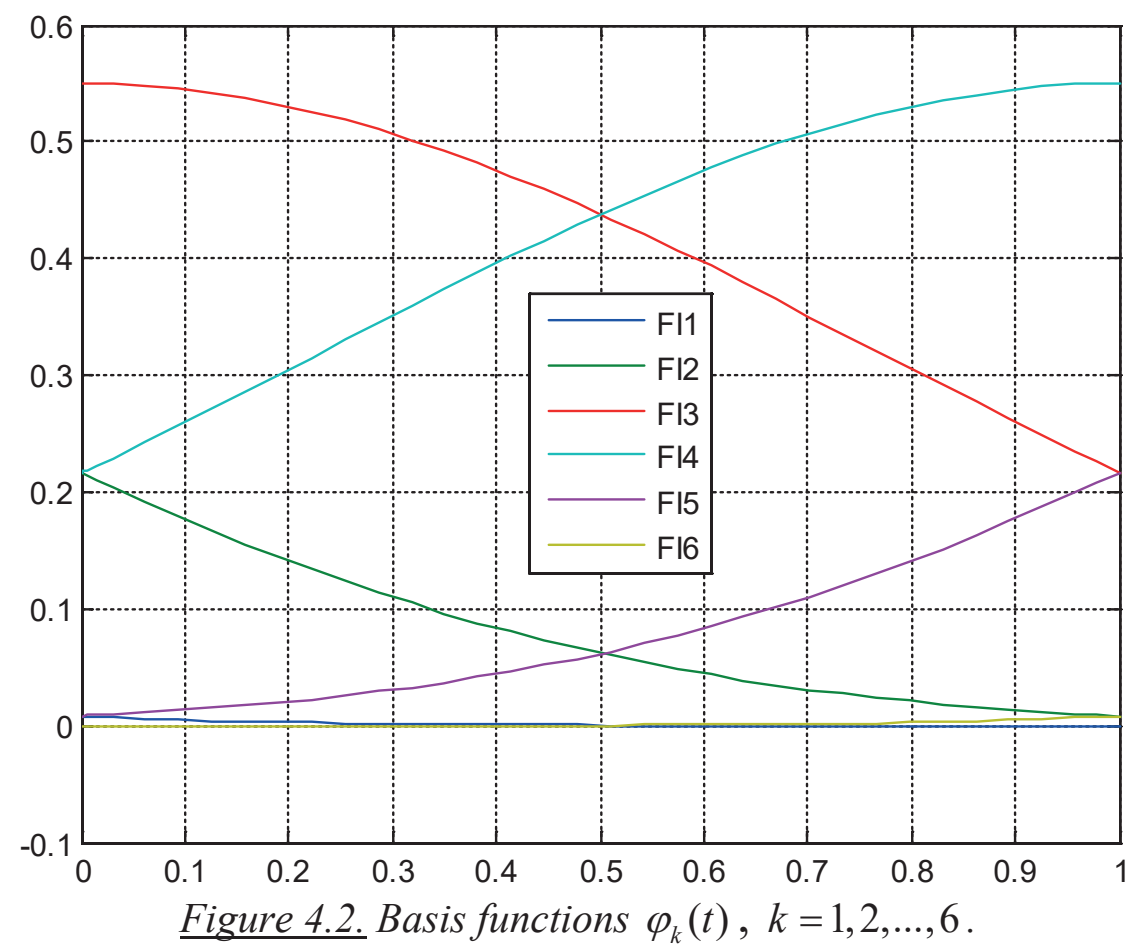

Since the number of unknowns on the element is equal to $N_{i e}=4$, we use a B-spline of the $\varphi(t)=\varphi_{0,2}(t+1)$ first degree in order to represent the unknown deflection function.

$\varphi(t)=\frac{1}{2} \Delta_{2}|t|=\frac{1}{2}[|t+1|-2|t|+|t-1|]$.

Let us use the following notation: 


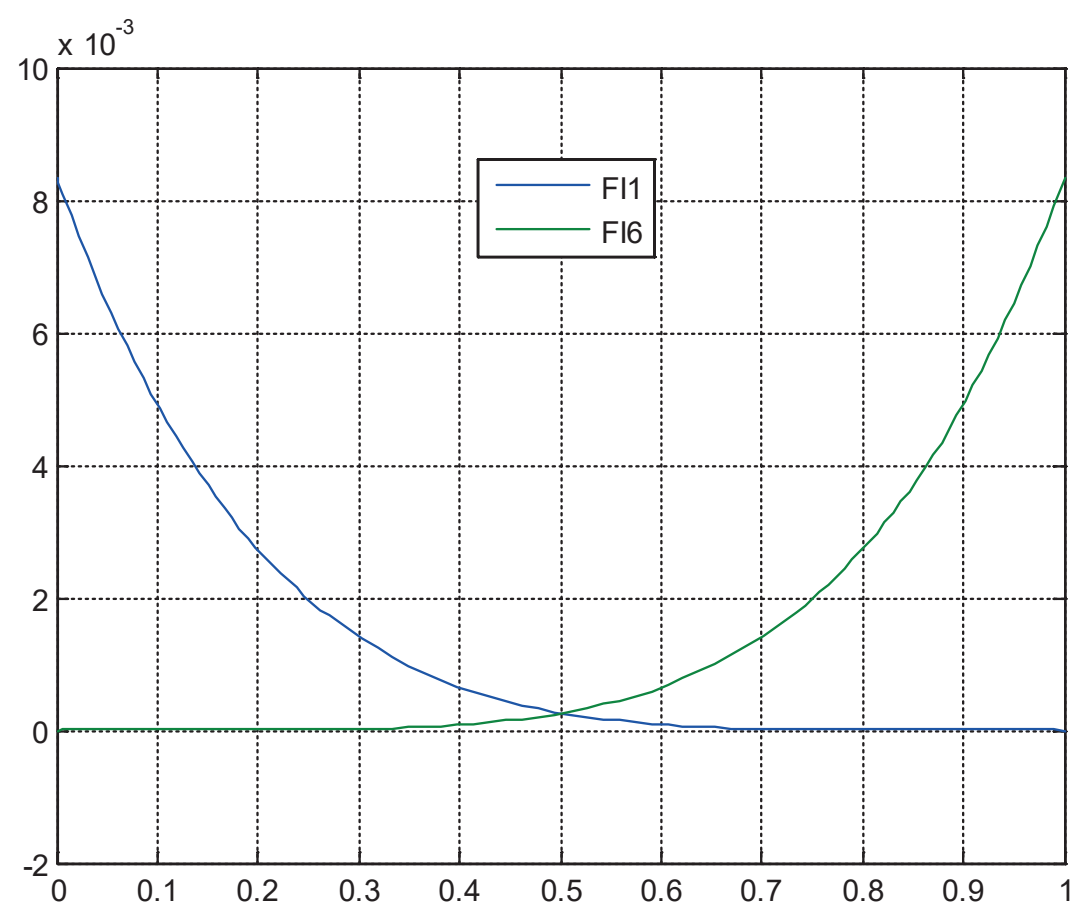

Figure 4.3. Basis functions $\varphi_{1}(t)$ and $\varphi_{6}(t)$.

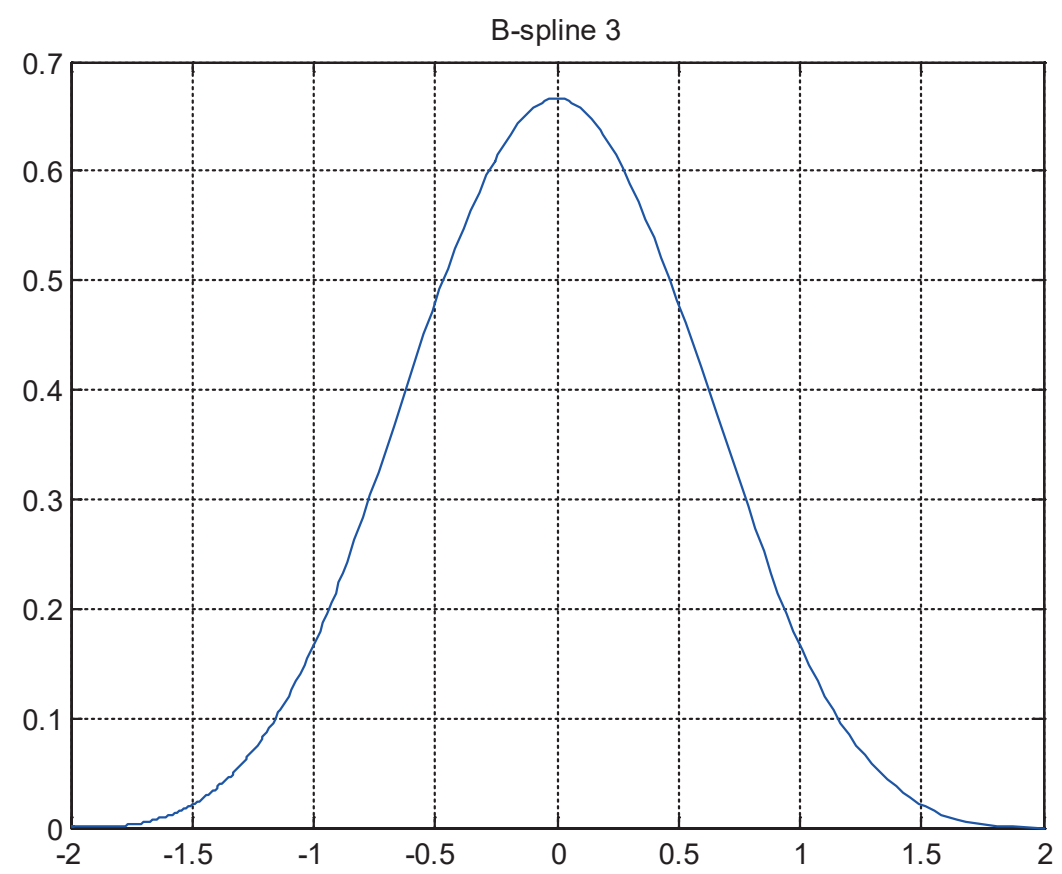

Figure 4.4. B-spline of the third order $\varphi(t)=\varphi_{0,4}(t+2)$.

This function is a B-spline, symmetric with respect to $t=0$ and its support is defined by an interval $[-1,1]$ (Figure 4.6).

We take the following four functions as basis functions on the unit interval (Figures 4.7):

$$
\varphi_{1}(t)=\varphi(t), \quad \varphi_{2}(t)=\varphi(t-1)
$$$$
0 \leq t \leq 1 \text {. (4.9) }
$$

We represent the unknown function $y(x)$ within the element number $i_{e}$ in the form 
Localization of Solution of the Problem of Two-Dimensional Theory of Elasticity with the Use of B-Spline DiscreteContinual Finite Element Method

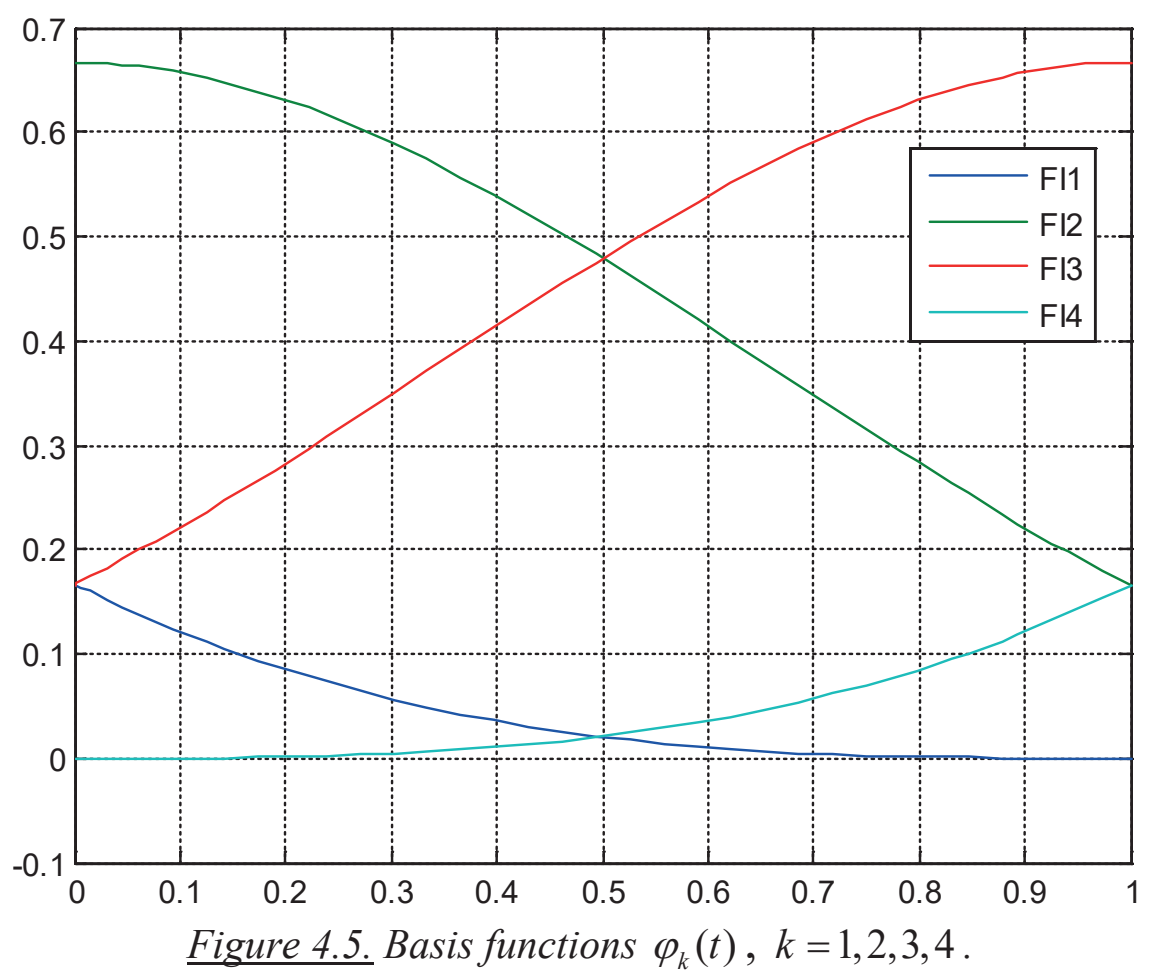

B-spline 1

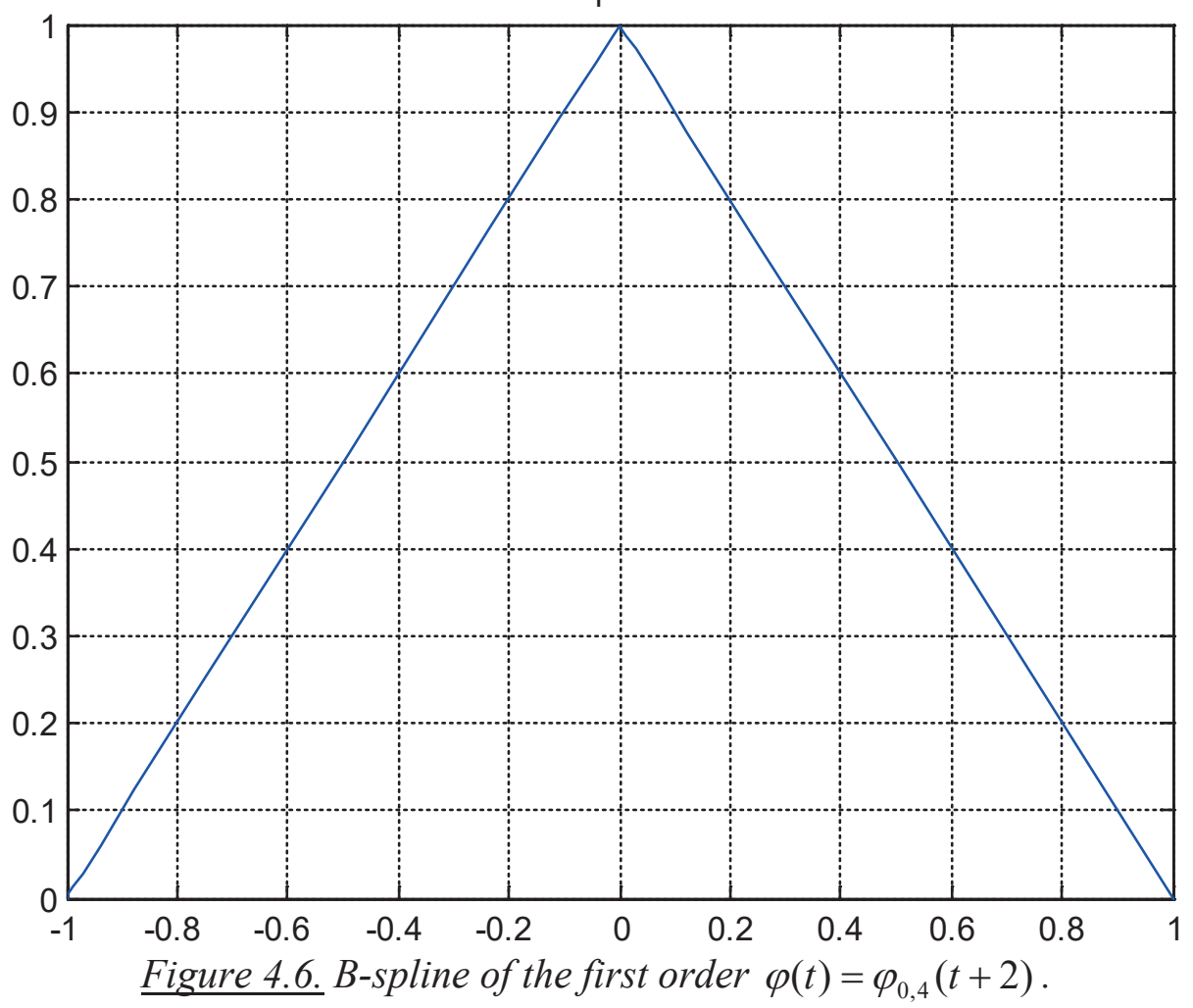

$y(x)=w(t)=\sum_{k=1}^{N_{p}} \alpha_{k} \varphi_{k}(t), \quad x_{1\left(i_{e}\right)} \leq x \leq x_{N_{p}\left(i_{e}\right)}$

We can define parameters $\alpha_{k}$ with the use of nodal unknowns of the element: 


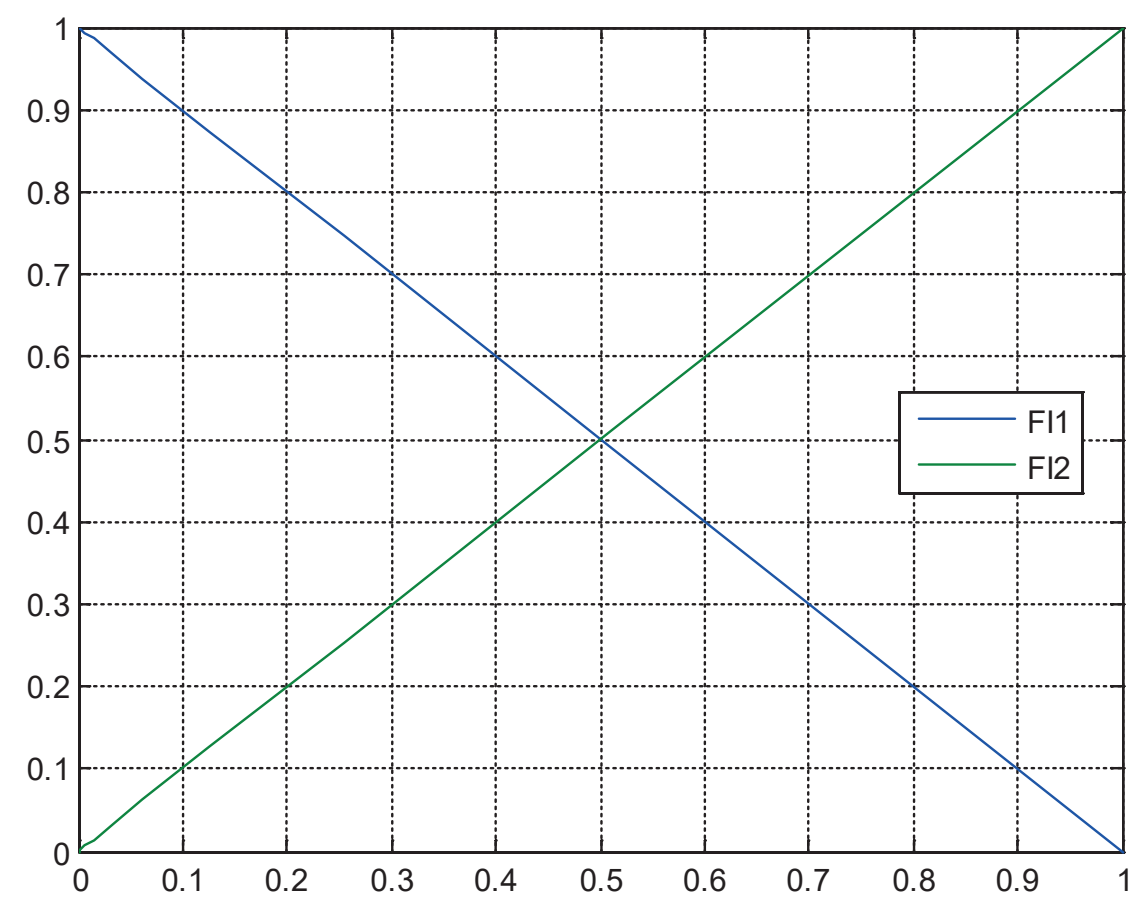

Figure 4.7. Basis functions $\varphi_{k}(t), k=1,2$.

$$
T_{6}=\left[\begin{array}{cccccc}
\varphi_{1}(0) & \varphi_{2}(0) & \varphi_{3}(0) & \varphi_{4}(0) & \varphi_{5}(0) & \varphi_{6}(0) \\
\varphi_{1}(0.2) & \varphi_{2}(0.2) & \varphi_{3}(0.2) & \varphi_{4}(0.2) & \varphi_{5}(0.2) & \varphi_{6}(0.2) \\
\varphi_{1}(0.4) & \varphi_{2}(0.4) & \varphi_{3}(0.4) & \varphi_{4}(0.4) & \varphi_{5}(0.4) & \varphi_{6}(0.4) \\
\varphi_{1}(0.6) & \varphi_{2}(0.6) & \varphi_{3}(0.6) & \varphi_{4}(0.6) & \varphi_{5}(0.6) & \varphi_{6}(0.6) \\
\varphi_{1}(0.8) & \varphi_{2}(0.8) & \varphi_{3}(0.8) & \varphi_{4}(0.8) & \varphi_{5}(0.8) & \varphi_{6}(0.8) \\
\varphi_{1}(1) & \varphi_{2}(1) & \varphi_{3}(1) & \varphi_{4}(1) & \varphi_{5}(1) & \varphi_{6}(1)
\end{array}\right]
$$

Figure 4.8. Matrix $T_{6}$.

$$
y_{i}=w\left(t_{i}\right)=\sum_{k=1}^{N_{p}} \alpha_{k} \varphi_{k}\left(t_{i}\right), \quad x_{1\left(i_{e}\right)} \leq x \leq x_{N_{p}\left(i_{e}\right)}, \quad T_{4}=\left[\begin{array}{cccc}
\varphi_{1}(0) & \varphi_{2}(0) & \varphi_{3}(0) & \varphi_{4}(0) \\
\varphi_{1}(1 / 3) & \varphi_{2}(1 / 3) & \varphi_{3}(1 / 3) & \varphi_{4}(1 / 3) \\
\varphi_{1}(2 / 3) & \varphi_{2}(2 / 3) & \varphi_{3}(2 / 3) & \varphi_{4}(2 / 3) \\
\varphi_{1}(1) & \varphi_{2}(1) & \varphi_{3}(1) & \varphi_{4}(1)
\end{array}\right] .
$$

In case $N_{p}=6$ we have (Figure 4.8)

$$
\begin{gathered}
\bar{y}^{i_{e}}=T_{6} \bar{\alpha}, \\
\bar{y}^{i_{e}}=\left[\begin{array}{llllll}
y_{1} & y_{2} & y_{3} & y_{4} & y_{5} & y_{6}
\end{array}\right]^{T} ; \\
\bar{\alpha}=\left[\begin{array}{llllll}
\alpha_{1} & \alpha_{2} & \alpha_{3} & \alpha_{4} & \alpha_{5} & \alpha_{6}
\end{array}\right]^{T} .
\end{gathered}
$$

In case $N_{p}=4$ we have

$$
\begin{gathered}
\bar{y}^{i_{e}}=T_{4} \bar{\alpha}, \\
\bar{y}^{i_{e}}=\left[\begin{array}{llll}
y_{1} & y_{2} & y_{3} & y_{4}
\end{array}\right]^{T} ; \\
\bar{\alpha}=\left[\begin{array}{llll}
\alpha_{1} & \alpha_{2} & \alpha_{3} & \alpha_{4}
\end{array}\right]^{T} ;
\end{gathered}
$$

In case $N_{p}=2$ we have

$$
\begin{gathered}
\bar{y}^{i_{e}}=T_{2} \bar{\alpha}, \\
\bar{y}^{i_{e}}=\left[\begin{array}{ll}
y_{1} & y_{2}
\end{array}\right]^{T} ; \quad \bar{\alpha}=\left[\begin{array}{ll}
\alpha_{1} & \alpha_{2}
\end{array}\right]^{T} ; \\
T_{2}=\left[\begin{array}{cc}
\varphi_{1}(0) & \varphi_{2}(0) \\
\varphi_{1}(1) & \varphi_{2}(1)
\end{array}\right] .
\end{gathered}
$$

Using (4.12)-(4.21), we get

$$
\bar{\alpha}=T_{N_{p}}^{-1} \bar{y}^{i_{e}},
$$


Localization of Solution of the Problem of Two-Dimensional Theory of Elasticity with the Use of B-Spline Discrete-

where

$$
\left[\begin{array}{l}
\bar{u}_{1}^{i e} \\
\bar{u}_{2}^{i e}
\end{array}\right]=P^{T} \bar{u}^{i e} .
$$

$$
T_{N_{p}}=\left\{T_{i j}\right\}_{i, j=1, \ldots, N_{p}} ; \quad T_{i j}=\varphi_{j}\left(t_{i}\right) .
$$

Let us introduce the following notation system:

$$
\bar{u}^{i e}=\left[\begin{array}{c}
\bar{u}^{1} \\
\vdots \\
\bar{u}^{N_{p}}
\end{array}\right]
$$

is nodal vector-function of element number $i_{e}$;

$$
\bar{u}^{i}=\left[\begin{array}{l}
u_{1}^{i} \\
u_{2}^{i}
\end{array}\right]
$$

is vector-unction in node number $i$ with the element number $i_{e}$;

$$
\bar{u}_{k}^{i e}=\left[\begin{array}{c}
u_{k}^{1} \\
\vdots \\
u_{k}^{N_{p}}
\end{array}\right], \quad k=1,2
$$

is nodal component number $k$ of vectorfunction of element number $i_{e}$.

Let $P$ be permutation matrix,

$$
\begin{aligned}
& \bar{u}^{i e}=P\left[\begin{array}{l}
\bar{u}_{1}^{i e} \\
\bar{u}_{2}^{i e}
\end{array}\right] ; \\
& P=\underbrace{\left[\begin{array}{cccc|cccc}
1 & 0 & \cdots & 0 & 0 & 0 & \cdots & 0 \\
0 & 0 & \cdots & 0 & 1 & 0 & \cdots & 0 \\
0 & 1 & \cdots & 0 & 0 & 0 & \cdots & 0 \\
0 & 0 & \cdots & 0 & 0 & 1 & \cdots & 0 \\
\vdots & \vdots & \cdots & \vdots & 0 & 0 & \cdots & 0 \\
\vdots & \vdots & \cdots & 0 & \vdots & \vdots & \cdots & \vdots \\
\vdots & \vdots & \cdots & 1 & \vdots & \vdots & \cdots & 0 \\
0 & 0 & \cdots & 0 & 0 & 0 & & 1
\end{array}\right] ;}_{2 \cdot N_{p}}
\end{aligned}
$$

Due to $P^{-1}=P^{T}$ we have
We have to consider bilinear forms with allowance for relations (4.2)-(4.3) in order to construct local stiffness matrices corresponding to the operators $L_{u u}, L_{u v}, L_{v v}$ (see (1.3)-(1.5)):

$$
\begin{aligned}
& \left(L_{u u} \bar{u}, \bar{z}\right)=\left(\partial^{*} D_{1}^{T} C D_{1} \partial \bar{u}, \bar{z}\right)=\left(D_{1}^{T} C D_{1} \partial \bar{u}, \partial \bar{z}\right)= \\
& =\int_{x_{1(i e)}}^{x_{N p(i)}}\left(\left[\begin{array}{cc}
2 \mu+\lambda & \mu
\end{array}\right] \partial \bar{u}, \partial \bar{z}\right) d x ;
\end{aligned}
$$

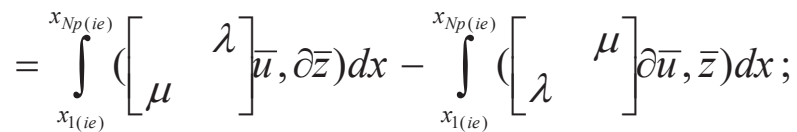

$$
\begin{aligned}
\left(L_{v v} \bar{u}, \bar{z}\right)= & \left(D_{2}^{T} C D_{2} \bar{u}, \bar{z}\right)= \\
& =\int_{x_{(i(i)}}^{x_{X_{p}(i)}}\left(\left[\begin{array}{ll}
\mu & \\
& 2 \mu+\lambda
\end{array}\right] \bar{u}, \bar{z}\right) d x
\end{aligned}
$$

for the following type of functions

$$
\begin{aligned}
y(x)=w(t)= & \sum_{j=1}^{N_{p}} \varphi_{j}(t) \bar{\alpha}^{j}, \\
& z(x)=\bar{q}(t)=\sum_{j=1}^{N_{p}} \varphi_{j}(t) \bar{\beta}^{j},
\end{aligned}
$$

where we have

$$
\begin{gathered}
x_{1(i e)} \leq x \leq x_{N_{p}(i e)}, \quad 0 \leq t \leq 1 \\
\bar{\alpha}^{j}=\left[\begin{array}{l}
\alpha_{1}^{j} \\
\alpha_{2}^{j}
\end{array}\right], \quad \bar{\beta}^{j}=\left[\begin{array}{l}
\beta_{1}^{j} \\
\beta_{2}^{j}
\end{array}\right], \quad j=1, \ldots, N_{p}
\end{gathered}
$$

Let us substitute (4.33) sequentially into (4.30)(4.32), changing the variable of integration (see (4.2)-(4.3)). Let us consider (4.30): 


$$
\begin{aligned}
& \int_{x_{1(i e)}}^{x_{N p(i e)}}\left(\left[\begin{array}{cc}
2 \mu+\lambda & \\
& \mu
\end{array}\right] \partial \bar{u}, \partial \bar{z}\right) d x \\
& =\frac{h_{e}}{h_{e}^{2}} \int_{0}^{1}\left(\left[\begin{array}{cc}
2 \mu+\lambda & \mu
\end{array}\right] \sum_{j=1}^{N_{p}} \varphi_{j}^{\prime}(t) \bar{\alpha}^{j}, \sum_{j=1}^{N_{p}} \varphi_{j}^{\prime}(t) \bar{\beta}^{j}\right) d t= \\
& =\sum_{i=1}^{N_{p}} \sum_{j=1}^{N_{p}}\left(\frac{1}{h_{e}} \int_{0}^{1} \varphi_{i}^{\prime}(t) \varphi_{j}^{\prime}(t) d t\left[\begin{array}{ll}
2 \mu+\lambda & \mu
\end{array}\right] \bar{\alpha}^{j}, \bar{\beta}^{i}\right)= \\
& \left.=\sum_{i=1}^{N_{p}} \sum_{j=1}^{N_{p}} b_{i j}\left(\begin{array}{ll}
2 \mu+\lambda & \\
& \mu
\end{array}\right] \bar{\alpha}^{j}, \bar{\beta}^{i}\right),
\end{aligned}
$$

where we have

$$
b_{i j}=\frac{1}{h_{e}} \int_{0}^{1} \varphi_{i}^{\prime}(t) \varphi_{j}^{\prime}(t) d t .
$$

It should be noted, in particular, that, $b_{j i}=b_{i j}$ i.e. if $B=\left\{b_{i j}\right\}_{i, j=1, \ldots, N_{p}}$, then $B^{T}=B$.

For further transformations, we use the representation

$$
\begin{gathered}
\bar{\alpha}^{j}=\left[\begin{array}{l}
\alpha_{1}^{j} \\
\alpha_{2}^{j}
\end{array}\right]=\alpha_{1}^{j}\left[\begin{array}{l}
1 \\
0
\end{array}\right]+\alpha_{2}^{j}\left[\begin{array}{l}
0 \\
1
\end{array}\right] ; \\
\bar{\beta}^{j}=\left[\begin{array}{l}
\beta_{1}^{j} \\
\beta_{2}^{j}
\end{array}\right]=\beta_{1}^{j}\left[\begin{array}{l}
1 \\
0
\end{array}\right]+\beta_{2}^{j}\left[\begin{array}{l}
0 \\
1
\end{array}\right] ; \\
\left.\left[\begin{array}{cc}
2 \mu+\lambda & \\
{\left[\begin{array}{cc}
2 \mu+\lambda &
\end{array}\right] \bar{\alpha}^{j}=\alpha_{1}^{j}(2 \mu+\lambda)\left[\begin{array}{l}
1 \\
0
\end{array}\right]+\alpha_{2}^{j} \mu\left[\begin{array}{l}
0 \\
1
\end{array}\right] ;}
\end{array}\right] \bar{\alpha}^{j}, \bar{\beta}^{i}\right)=(2 \mu+\lambda) \alpha_{1}^{j} \beta_{1}^{i}+\mu \alpha_{2}^{j} \beta_{2}^{i} .
\end{gathered}
$$

We can substitute (4.37) in (4.35). Taking into account (4.22)-(4.29) and the adopted notation we get

$$
\begin{aligned}
& \sum_{i=1}^{N_{p}} \sum_{j=1}^{N_{p}} b_{i j}\left((2 \mu+\lambda) \alpha_{1}^{j} \beta_{1}^{i}+\mu \alpha_{2}^{j} \beta_{2}^{i}\right)= \\
& \quad=(2 \mu+\lambda) \sum_{i=1}^{N_{p}} \sum_{j=1}^{N_{p}} b_{i j} \alpha_{1}^{j} \beta_{1}^{i}+\mu \sum_{i=1}^{N_{p}} \sum_{j=1}^{N_{p}} b_{i j} \alpha_{2}^{j} \beta_{2}^{i}=
\end{aligned}
$$

$$
\begin{gathered}
=(2 \mu+\lambda)\left(B \bar{\alpha}_{1}, \bar{\beta}_{1}\right)+\mu\left(B \bar{\alpha}_{2}, \bar{\beta}_{2}\right)= \\
=(2 \mu+\lambda)\left(B T_{N_{p}}^{-1} \bar{u}_{1}^{i e}, T_{N_{p}}^{-1} \bar{z}_{1}^{i e}\right)+ \\
+\mu\left(B T_{N_{p}}^{-1} \bar{u}_{2}^{i e}, T_{N_{p}}^{-1} \bar{z}_{2}^{i e}\right)= \\
=(2 \mu+\lambda)\left(\left(T_{N_{p}}^{-1}\right)^{T} B T_{N_{p}}^{-1} \bar{u}_{1}^{i e}, \bar{z}_{1}^{i e}\right)+ \\
+\mu\left(\left(T_{N_{p}}^{-1}\right)^{T} B T_{N_{p}}^{-1} \bar{u}_{2}^{i e}, \bar{z}_{2}^{i e}\right)= \\
=(2 \mu+\lambda)\left(A_{11} \bar{u}_{1}^{i e}, \bar{z}_{1}^{i e}\right)+\mu\left(A_{11} \bar{u}_{2}^{i e}, \bar{z}_{2}^{i e}\right)= \\
=\left(\left[\begin{array}{c|c}
(2 \mu+\lambda) A_{11} & 0 \\
\hline 0 & 0
\end{array}\right]\left[\begin{array}{l}
\bar{u}_{1}^{i e} \\
\bar{u}_{2}^{i e}
\end{array}\right],\left[\begin{array}{l}
\bar{z}_{1}^{i e} \\
\bar{z}_{2}^{i e}
\end{array}\right]\right)+ \\
+\left(\left[\begin{array}{c|c}
0 & 0 \\
\hline 0 & \mu A_{11}
\end{array}\right]\left[\begin{array}{l}
\bar{u}_{1}^{i e} \\
\bar{u}_{2}^{i e}
\end{array}\right],\left[\begin{array}{c}
\bar{z}_{1}^{i e} \\
\bar{z}_{2}^{i e}
\end{array}\right]\right)= \\
=\left(\left[\begin{array}{c|c}
(2 \mu+\lambda) A_{11} & 0 \\
\hline 0 & \mu A_{11}
\end{array}\right]\left[\begin{array}{l}
\bar{u}_{1}^{i e} \\
\bar{u}_{2}^{i e}
\end{array}\right],\left[\begin{array}{c}
\bar{z}_{1}^{i e} \\
\bar{z}_{2}^{i e}
\end{array}\right]\right)= \\
\left.=\left(\begin{array}{c|c}
(2 \mu+\lambda) A_{11} & 0 \\
\hline 0 & \mu A_{11}
\end{array}\right] P^{T} \bar{u}^{i e}, P^{T} \bar{z}^{i e}\right)= \\
\left.\hline\left[\begin{array}{c|c}
(2 \mu+\lambda) A_{11} & 0 \\
\hline 0 & \mu A_{11}
\end{array}\right] P^{T} \bar{u}^{i e}, \bar{z}^{i e}\right)=
\end{gathered}
$$

Thus, an expression is obtained for the local stiffness matrix corresponding to the operator $L_{u u}$ within the element number $i_{e}$ :

$$
K_{u u}^{i e}=P\left[\begin{array}{c|c}
(2 \mu+\lambda) A_{11} & 0 \\
\hline 0 & \mu A_{11}
\end{array}\right] P^{T},
$$

where we have

$$
A_{11}=\left(T_{N_{p}}^{-1}\right)^{T} B T_{N_{p}}^{-1}
$$

Then we can consider (4.31) in a similar way:

$$
\begin{aligned}
& \left.\left.\int_{x_{1(i e)}}^{x_{N p(i e)}}\left(\mu^{\mu}\right] \bar{u}, \partial \bar{z}\right) d x-\int_{x_{1(i e)}}^{x_{N p}(i e)}\left[\lambda^{\lambda}\right] \partial \bar{u}, \bar{z}\right) d x=
\end{aligned}
$$

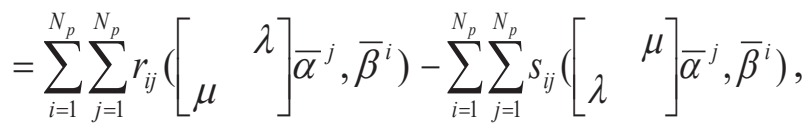

where we have 
Localization of Solution of the Problem of Two-Dimensional Theory of Elasticity with the Use of B-Spline DiscreteContinual Finite Element Method

$$
r_{i j}=\int_{0}^{1} \varphi_{i}^{\prime}(t) \varphi_{j}(t) d t ; \quad s_{i j}=\int_{0}^{1} \varphi_{i}(t) \varphi_{j}^{\prime}(t) d t .
$$

We should note that if

$$
R=\left\{r_{i j}\right\}_{i, j=1, \ldots, N_{p}}, \quad S=\left\{S_{i j}\right\}_{i, j=1, \ldots, N_{p}},
$$

we get

$$
R^{T}=S
$$

Let us define the elements of the sums (4.41):

$$
\begin{aligned}
& {\left[\begin{array}{ll}
\mu & \lambda
\end{array}\right] \bar{\alpha}^{j}=\alpha_{2}^{j} \lambda\left[\begin{array}{l}
1 \\
0
\end{array}\right]+\alpha_{1}^{j} \mu\left[\begin{array}{l}
0 \\
1
\end{array}\right] ;} \\
& \left(\left[\begin{array}{ll}
\mu & \lambda
\end{array} \bar{\alpha}^{j}, \bar{\beta}^{i}\right)=\mu \alpha_{1}^{j} \beta_{2}^{i}+\lambda \alpha_{2}^{j} \beta_{1}^{i} ;\right. \\
& {\left[\begin{array}{ll}
\lambda & \mu
\end{array}\right] \bar{\alpha}^{j}=\alpha_{2}^{j} \mu\left[\begin{array}{l}
1 \\
0
\end{array}\right]+\alpha_{1}^{j} \lambda\left[\begin{array}{l}
0 \\
1
\end{array}\right] ;} \\
& \left(\left[\begin{array}{ll}
\lambda & \mu
\end{array}\right] \bar{\alpha}^{j}, \bar{\beta}^{i}\right)=\lambda \alpha_{1}^{j} \beta_{2}^{i}+\mu \alpha_{2}^{j} \beta_{1}^{i} \text {. }
\end{aligned}
$$

Substituting (4.43) into (4.41) and, taking into account (4.22)-(4.29) and the accepted notation, we obtain

$$
\begin{aligned}
& \sum_{i=1}^{N_{p}} \sum_{j=1}^{N_{p}} s_{i j}\left(\lambda \alpha_{1}^{j} \beta_{2}^{i}+\mu \alpha_{2}^{j} \beta_{1}^{i}\right)= \\
& =\lambda \sum_{i=1}^{N_{p}} \sum_{j=1}^{N_{p}} s_{i j} \alpha_{1}^{j} \beta_{2}^{i}+\mu \sum_{i=1}^{N_{p}} \sum_{j=1}^{N_{p}} s_{i j} \alpha_{2}^{j} \beta_{1}^{i}= \\
& =\lambda\left(S \bar{\alpha}_{1}, \bar{\beta}_{2}\right)+\mu\left(S \bar{\alpha}_{2}, \bar{\beta}_{1}\right)= \\
& =\lambda\left(S T_{N_{p}}^{-1} \bar{u}_{1}^{i e}, T_{N_{p}}^{-1} \bar{z}_{2}^{i e}\right)+\mu\left(S T_{N_{p}}^{-1} \bar{u}_{2}^{i e}, T_{N_{p}}^{-1} \bar{z}_{1}^{i e}\right)= \\
& =\lambda\left(\left(T_{N_{p}}^{-1}\right)^{T} S T_{N_{p}}^{-1} \bar{u}_{1}^{i e}, \bar{z}_{2}^{i e}\right) \\
& +\mu\left(\left(T_{N_{p}}^{-1}\right)^{T} S T_{N_{p}}^{-1} \bar{u}_{2}^{i e}, \bar{z}_{1}^{i e}\right)=\lambda\left(A_{01} \bar{u}_{1}^{i e}, \bar{z}_{2}^{i e}\right)+ \\
& +\mu\left(A_{01} \bar{u}_{2}^{i e}, \bar{z}_{1}^{i e}\right)= \\
& =\left(\left[\begin{array}{c|c}
0 & \mu A_{01} \\
\hline \lambda A_{01} & 0
\end{array}\right]\left[\begin{array}{c}
\bar{u}_{1}^{i e} \\
\bar{u}_{2}^{i e}
\end{array}\right],\left[\begin{array}{c}
\bar{z}_{1}^{i e} \\
\bar{z}_{2}^{i e}
\end{array}\right]\right)= \\
& \left.=\left(\begin{array}{c|c}
P & \mu A_{01} \\
\hline \lambda A_{01} & 0
\end{array}\right] P^{T} \bar{u}^{i e}, \bar{z}^{i e}\right)= \\
& =\left(K_{2, u v}^{i e} \bar{u}^{i e}, \bar{z}^{i e}\right) \text {. }
\end{aligned}
$$

Thus, an expression is obtained for the local stiffness matrix corresponding to the operator $L_{u v}$ within the element number $i_{e}$ :

$$
K_{u v}^{i e}=K_{1, u v}^{i e}-K_{2, u v}^{i e},
$$

where we have

$$
K_{1, u v}^{i e}=P\left[\begin{array}{c|c}
0 & \lambda A_{10} \\
\hline \mu A_{10} & 0
\end{array}\right] P^{T} ; \quad A_{10}=\left(T_{N_{p}}^{-1}\right)^{T} R T_{N_{p}}^{-1} ;
$$

$$
K_{2, u v}^{i e}=P\left[\begin{array}{c|c}
0 & \mu A_{01} \\
\hline \lambda A_{01} & 0
\end{array}\right] P^{T} ; \quad A_{01}=\left(T_{N_{p}}^{-1}\right)^{T} S T_{N_{p}}^{-1} .
$$

Let us note that

$$
K_{2, u v}^{i e}=\left(K_{1, u v}^{i e}\right)^{T} .
$$

Let us further consider (4.32) in a similar way:

$$
\begin{aligned}
& \left.\int_{x_{1(i e)}}^{x_{N_{p}(i e)}}\left(\begin{array}{ll}
\mu & \\
= & 2 \mu+\lambda
\end{array}\right] \bar{\mu}, \bar{z}\right) d x= \\
& \left.h_{e} \int_{0}^{1}\left[\begin{array}{ll}
\mu & 2 \mu+\lambda
\end{array}\right] \sum_{j=1}^{N_{p}} \varphi_{j}(t) \bar{\alpha}^{j}, \sum_{j=1}^{N_{p}} \varphi_{j}(t) \bar{\beta}^{j}\right) d t= \\
& =\sum_{i=1}^{N_{p}} \sum_{j=1}^{N_{p}}\left(h_{e} \int_{0}^{1} \varphi_{i}(t) \varphi_{j}(t) d t\left[\begin{array}{ll}
\mu & \\
& 2 \mu+\lambda
\end{array}\right] \bar{\alpha}^{j}, \bar{\beta}^{i}\right)= \\
& \left.=\sum_{i=1}^{N_{p}} \sum_{j=1}^{N_{p}} m_{i j}\left[\begin{array}{ll}
\mu & 2 \mu+\lambda
\end{array}\right] \bar{\alpha}^{j}, \bar{\beta}^{i}\right), \quad(4.50)
\end{aligned}
$$

where we have

$$
m_{i j}=h_{e} \int_{0}^{1} \varphi_{i}(t) \varphi_{j}(t) d t .
$$

We should note that, in particular $m_{j i}=m_{i j}$, i.e. if $M=\left\{m_{i j}\right\}_{i, j=1, \ldots, N_{p}}$ we get

$$
M^{T}=M .
$$

For further transformations, we use the representation 
$\left(\left[\begin{array}{ll}\mu & \\ & 2 \mu+\lambda\end{array}\right] \bar{\alpha}^{j}, \bar{\beta}^{i}\right)=\mu \alpha_{1}^{j} \beta_{1}^{i}+(2 \mu+\lambda) \alpha_{2}^{j} \beta_{2}^{i}$.

We can substitute (4.52) in (4.50) and taking into account (4.22)-(4.29) and the adopted notation we get:

$$
\begin{gathered}
\sum_{i=1}^{N_{p}} \sum_{j=1}^{N_{p}} m_{i j}\left(\mu \alpha_{1}^{j} \beta_{1}^{i}+(2 \mu+\lambda) \alpha_{2}^{j} \beta_{2}^{i}\right)= \\
=\mu \sum_{i=1}^{N_{p}} \sum_{j=1}^{N_{p}} m_{i j} \alpha_{1}^{j} \beta_{1}^{i}+(2 \mu+\lambda) \sum_{i=1}^{N_{p}} \sum_{j=1}^{N_{p}} m_{i j} \alpha_{2}^{j} \beta_{2}^{i}= \\
=\mu\left(M \alpha_{1}, \bar{\beta}_{1}\right)+(2 \mu+\lambda)\left(M \bar{\alpha}_{2}, \bar{\beta}_{2}\right)= \\
=\mu\left(\left(T_{N_{p}}^{-1}\right)^{T} M T_{N_{p}}^{-1} \bar{u}_{1}^{i e}, \bar{z}_{1}^{i e}\right)+ \\
+(2 \mu+\lambda)\left(\left(T_{N_{p}}^{-1}\right)^{T} B T_{N_{p}}^{-1} \bar{u}_{2}^{i e}, \bar{z}_{2}^{i e}\right)= \\
=\mu\left(A_{00} \bar{u}_{1}^{i e}, \bar{z}_{1}^{i e}\right)+(2 \mu+\lambda)\left(A_{00} \bar{u}_{2}^{i e}, \bar{z}_{2}^{i e}\right)= \\
=\left(P\left[\begin{array}{c|c}
\mu A_{00} & 0 \\
\hline 0 & (2 \mu+\lambda) A_{00}
\end{array}\right] P^{T} \bar{u}^{i e}, \bar{z}^{i e}\right)= \\
=\left(K_{v v}^{i e} \bar{u}^{i e}, \bar{z}^{i e}\right) . \quad(4.53)
\end{gathered}
$$

Thus, an expression is obtained for the local stiffness matrix corresponding to the operator $L_{v}$ within the element number $i_{e}$ :

$$
K_{u u}^{i e}=P\left[\begin{array}{c|c}
\mu A_{00} & 0 \\
\hline 0 & (2 \mu+\lambda) A_{00}
\end{array}\right] P^{T},
$$

where we have

$$
A_{00}=\left(T_{N_{p}}^{-1}\right)^{T} M T_{N_{p}}^{-1}
$$

\section{SEVERAL ASPECTS \\ OF NUMERICAL IMPLEMENTATION}

The presented algorithm can be implemented using MATLAB tools. The MATLAB system has convenient functions for working with polynomials. Moreover, the main parameter of these functions is the vector of coefficients of the polynomial. To determine the coefficients of basic polynomials $\varphi_{k}$ on an interval [ $\left[\begin{array}{ll}0 & 1\end{array}\right]$, we can firstly determine their values at $N_{p}$ points of the interval $t=\left[t_{1}, t_{2}, \ldots, t_{N_{p}}\right], \quad t_{i} \in\left[\begin{array}{ll}0 & 1\end{array}\right]$, $i=1,2, \ldots, N_{p}$ :

$$
F_{k}(i)=\varphi_{k}\left(t_{i}\right), \quad i=1,2, \ldots, N_{p}, \quad k=1,2, \ldots, N_{p} .
$$

Then, using the polyfit function, we define their coefficient vector $p_{k}$ :

$$
\mathrm{pk}=\mathrm{polyfit}(\mathrm{t}, \mathrm{Fk}, \mathrm{Nk})
$$

This function is used to determine the coefficients of the optimal polynomial using the least squares method. In the considering case, we construct polynomial of the $\left(N_{p}-1\right)$ th degree (i.e. we have to define $N_{p}$ coefficients of polynomial, according to its $N_{p}$ values), therefore, we get a polynomial passing through the given values.

In order to calculate the derivatives we can sequentially use the polyder function:

$$
\mathrm{dpk}=\mathrm{pol} \text { yder }(\mathrm{pk})
$$

is the vector of coefficients $\varphi_{k}^{\prime}$.

In order to calculate the product of polynomials we can use the conv function:

$$
\mathrm{pij}=\mathrm{conv}(\mathrm{p} i, \mathrm{pj})
$$

is the vector of coefficients $\varphi_{i} \varphi_{j}$;

$$
\text { d10pij=conv (dpi, pj) }
$$

is the vector of coefficients $\varphi_{i}^{\prime} \varphi_{j}$;

$$
\mathrm{d} 01 \mathrm{pij}=\mathrm{conv}(\mathrm{pi}, \mathrm{dpj})
$$

is the vector of coefficients $\varphi_{i} \varphi_{j}^{\prime}$;

$$
\text { dpij=conv (dpi, dpj) }
$$

is the vector of coefficients $\varphi_{i}^{\prime} \varphi_{j}^{\prime}$.

In order to calculate the antiderivative of a polynomial we can use the polyint function:

$$
\text { Pi=polyint (pi) }
$$

is the vector of coefficients $\int \varphi_{i} d t$; 


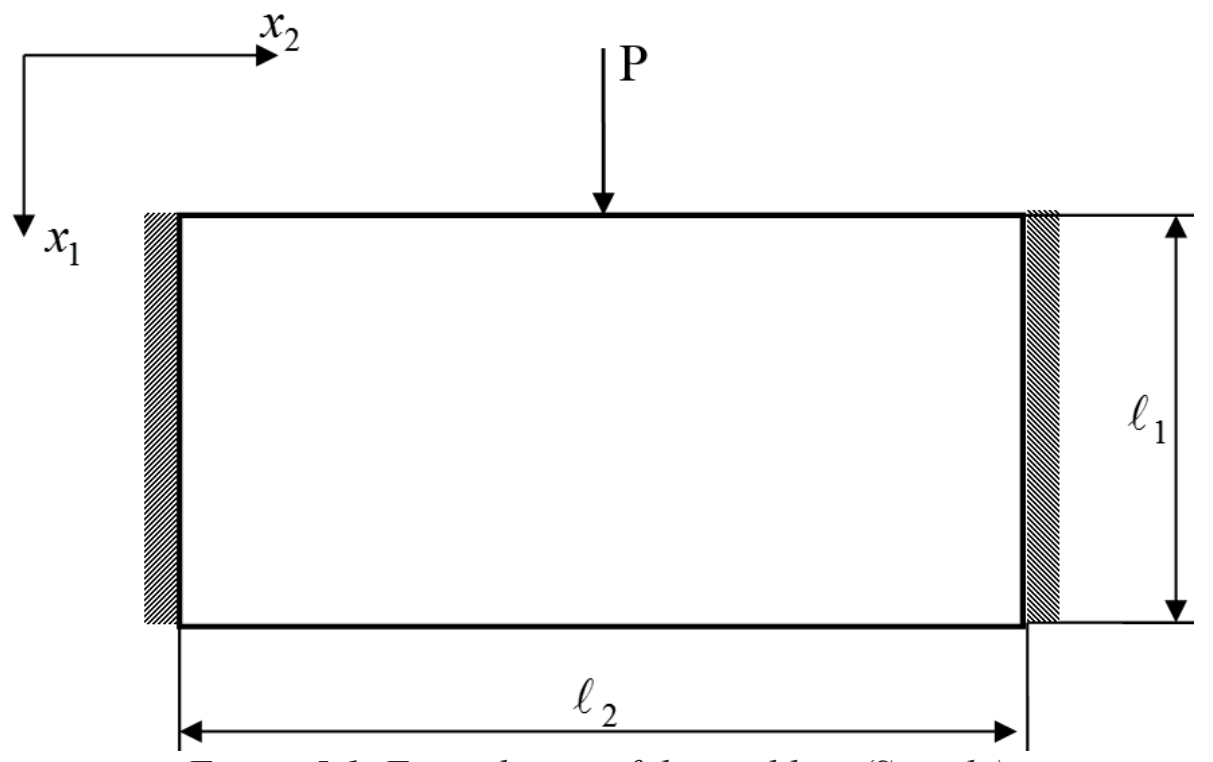

Figure 5.1. Formulation of the problem (Sample).

Pij=polyint (pij)

is the vector of coefficients $\int \varphi_{i} \varphi_{j} d t$;

d10Pij=polyint (d10pij)

is the vector of coefficients $\int \varphi_{i}^{\prime} \varphi_{j} d t$;

d01Pij=polyint (d01pij)

is the vector of coefficients $\int \varphi_{i} \varphi_{j}^{\prime} d t$;

$$
\mathrm{dPij}=\mathrm{polyint}(\mathrm{dp} i j)
$$

is the vector of coefficients $\int \varphi_{i}^{\prime} \varphi_{j}^{\prime} d t$;

Then the calculation of

$$
B(i, j), \quad R(i, j), \quad S(i, j), \quad M(i, j)
$$

can be done in accordance with formulas

$$
\begin{aligned}
& M(i, j)=\text { he[polyval }(\mathrm{Pij}, 1)- \\
& \text { polyval (Pij,0)]; } \\
& R(i, j)=\text { polyval }(\mathrm{d} 10 \mathrm{Pij}, 1)- \\
& \text { polyval (d10Pij,0); } \\
& S(i, j)=\operatorname{polyval}(\mathrm{d} 01 \mathrm{Pij}, 1)- \\
& \text { polyval (d01Pij, 0); } \\
& B(i, j)=\text { [polyval }(\mathrm{dPij}, 1)- \\
& \text { polyval (dPij, 0) ] /he, }
\end{aligned}
$$

where the function polyval (,$t$ ) allows researcher to calculate the values of a polynomial with a vector of coefficients $p$ at a given point $t$.

\section{EXAMPLE OF ANALYSIS}

\subsection{Formulation of the problem.}

As a model example, let us consider the determination of the displacements of a beam-wall, fixed along the side faces in both directions, under the influence of a load concentrated in the center (Figure 5.1).

Let us consider the following geometric parameters: $\ell_{1}=6 \mathrm{~m}, \ell_{2}=12 \mathrm{~m}$.

Let us consider the following design parameters of material of plate: coefficient of elasticity $E=26500 \cdot 10^{4} \mathrm{kN} / \mathrm{m}^{2}$, Poisson's ratio $v=0.15$. Let external load parameter be equal to $P=100 \mathrm{kN}$.

\subsection{Structural analysis with allowance for localization.}

Let the number of elements be equal to $N_{e}=6$.

Then we have the following element length:

$$
h_{e}=\ell_{1} / N_{e}=6 / 4=1.5 \text {. }
$$

Let's define localization in the load area.

For the first element we have $N_{k}=5$ and fifthorder spline; distance between the coordinates of the nodes of the first element is equal to $h_{1}=1.5 / 5=0.3$. 


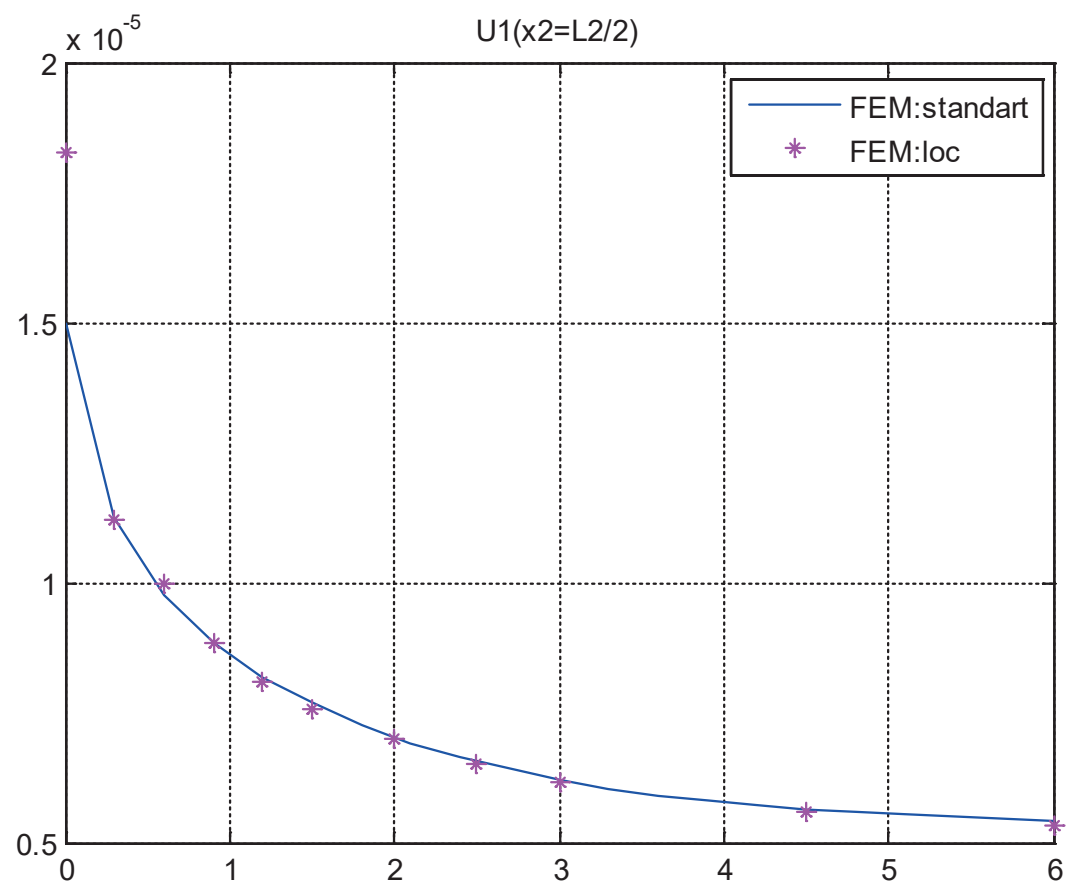

Figures 5.2. Comparison of the results of analysis in the middle sections along $x_{1}$ direction (discrete direction).

For the second element we have $N_{k}=3$ and third-order spline; distance between the coordinates of the nodes of the second element is equal to $h_{2}=1.5 / 3=0.5$.

For the third element and for the fourth element we have $N_{k}=1$ and first-order spline; distance between the coordinates of the nodes of the third element and of the fourth element is equal to $h_{3}=h_{4}=1.5 / 5=0.3$.

With such approximation the total number of nodes for all elements is equal to

$$
N_{x}=5+3+2 \cdot 1+1=11 .
$$

Then the total number of unknown nodal values for vectors $\bar{u}$ and $\bar{v}=\bar{u}^{\prime}$ is equal to

$$
N_{g}=4 \cdot N_{x}=4 \cdot 11=44 .
$$

\subsection{Structural analysis without localization.} In this case, we will consider only the standard linear fulfilment. In this case, the length of the element is taken equal to the minimum distance between the nodes, i.e. $h_{e}=0.3$. Then the number of elements is equal to

$$
N_{e}=6 / 0.3=20
$$

and the total number of nodes is equal to $N_{x}=21$. In this case, the number of nodal unknowns for each component of the vectors $\bar{u}$ and $\bar{v}=\bar{u}^{\prime}$ is equal to

$$
N_{g}=4 \cdot N_{x}=4 \cdot 21=84 .
$$

Graphical comparison of corresponding results of analysis is presented at Figure 5.2 and Figure 5.3 (FEM: loc-spline are nodal values computed with allowance for localization; FEM:standart are nodal values computed without localization).

As researcher can see, the results obtained are almost completely identical. Besides, the use of localization based on application of B-splines of various degrees leads to a significant decrease in the number of unknowns. The difference for this example is equal to $\Delta=84-44=40$. 


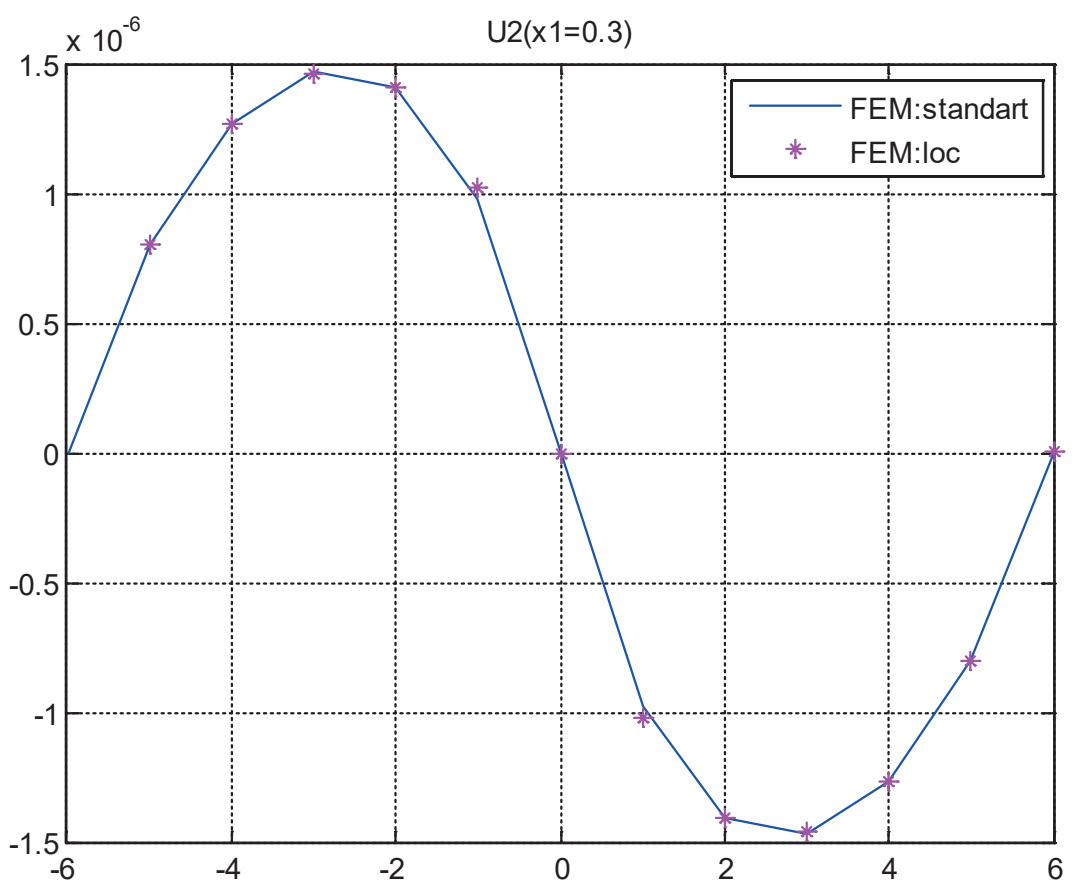

Figures 5.3. Comparison of the results of analysis in the middle sections along $x_{2}$ direction (continual direction).

\section{REFERENCES}

1. Akimov P.A., Mozgaleva M.L., Kaytukov T.B. Numerical solution of the problem of isotropic plate analysis with the use of B-spline discrete-continual finite element method. // // International Journal for Computational Civil and Structural Engineering, 2020, Vol. 16, Issue 4, pp. 14-28.

2. Akimov P.A., Mozgaleva M.L., Kaytukov T.B. Numerical solution of the problem of isotropic plate analysis with the use of B-spline discrete-continual finite element method. // // International Journal for Computational Civil and Structural Engineering, 2020, Vol. 16, Issue 4, pp. 14-28.

3. Akimov P.A., Mozgaleva M.L., Kaytukov T.B. Numerical solution of the problem of beam analysis with the use of B-spline finite element method. // International Journal for Computational Civil and Structural Engineering, 2020, Vol. 16, Issue 3, pp. 12-22.

4. Li B., Chen X. Wavelet-based numerical analysis: A review and classification. // Finite
Elements in Analysis and Design, 2014, Vol. 81, pp. 14-31.

5. Daubechies I. Orthonormal bases of compactly supported wavelets. // Commun. Pure Appl. Math., 1988, Vol. 41, pp. 909-996.

6. Li B., Cao H.R., He Z.J. The construction of one-dimensional Daubechies waveletbased finite elements for structural response analysis. // J. Vibroeng, 2011, vol. 13, pp. 729-738.

7. Ma J.R., Xue J.J. A study of the construction and application of a Daubechies wave-letbased beam element. // Finite Elements in Analysis and Design, 2003, Vol. 39, pp. 965-975.

8. Mozgaleva M.L., Akimov P.A., Kaytukov T.B. About wavelet-based computational beam analysis with the use of Daubechies scaling functions. // International Journal for Computational Civil and Structural Engineering, 2019, Vol. 15, Issue 2, pp. 95-110.

9. Mozgaleva M.L., Akimov P.A., Kaytukov

T.B. Wavelet-based discrete-continual finite element plate analysis with the use of 
Daubechies scaling functions. // International Journal for Computational Civil and Structural Engineering, 2019, Vol. 15, Issue 3, pp. 96-108.

10. Antes H. Bicubic fundamental splines in plate bending. // Int. J. Numer. Methods Eng., 1974, Vol. 8, pp. 503-511.

11. Han J.G., Ren W.X., Huang Y. A spline wavelet finite-element method in structural mechanics. // Int. J. Numer. Methods Eng., 2006, Vol. 66, pp. 166-190.

12. Han J.G., Ren W.X., Huang Y. A spline wavelet finite element formulation of thin plate bending. // Eng. Comput., 2009, Vol. 25, pp. 319-326.

13. Xiang J.W., Chen X.F., He Z.J., Zhang Y.H. A new wavelet-based thin plate element using B-spline wavelet on the interval. // Comput. Math., 2008, Vol. 41, pp. 243-255.

14. Chen X.F., Xiang J.W., Li B., He Z.J. A study of multiscale wavelet-based elements for adaptive finite element analysis. // Adv. Eng. Softw., 2010, Vol. 41, pp. 196-205.

15. Zhong Y.T., Xiang J.W. Construction of wavelet-based elements for static and stability analysis of elastic problems. // Acta Mech. Solida Sin., 2011, Vol. 24, pp. 355-364.

16. Yang Z.B., Chen X.F., Li B., He Z.J., Miao H.H. Vibration analysis of curved shell using b-spline wavelet on the interval (BSWI). // Finite Elements Method and General Shell Theory, CMES85, 2012, pp. 129-155.

17. Yang Z.B., Chen X.F., Zhang X.W., He Z.J. Free vibration and buckling analysis of plates using B-spline wavelet on the interval Mindlin element. // Appl. Math. Model., 2013, Vol. 37, pp. 3449-3466.

18. Xiang J.W., Chen X.F., Li B., He Y.M., He Z.J. Identification of a crack in a beam based on the finite element method of a B-spline wavelet on the interval. // J. Sound Vibr., 2006, Vol. 296, pp. 1046-1052.

19. Xiang J.W., Chen X.F., Mo Q.M., He Z.H. Identification of crack in a rotor system based on wavelet finite element method. // Finite Elem. Anal. Des., 2007, Vol. 43, pp. 1068-1081.
20. Xiang J.W., Liang M. A two-step approach to multi-damage detection for plate structures. // Eng. Fract. Mech., 2012, Vol. 91, pp. 73-86.

21. Xiang J.W., Matsumoto T., Long J.Q., Ma G. Identification of damage locations based on operating deflection shape. // Nondestruct. Test. Eval., 2012, Vol. 1, pp. 1-15.

22. Xiang J.W., Liang M. Wavelet-based detection of beam cracks using modal shape and frequency measurements. // Comput.Aided Civil Infrastruct. Eng., 2012, Vol. 27, pp. 439-454.

23. Xiang J.W., Matsumoto T., Wang Y.W., Jiang Z.S. Detect damages in conical shells using curvature mode shape and wavelet finite element method. // Int. J. Mech. Sci., 2013, Vol. 66, pp. 83-93.

24. Dong H.B., Chen X.F., Li B., Qi K.Y., He Z.J. Rotor crack detection based on highprecision modal parameter identification method and wavelet finite element model. // Mech. Syst. Signal Process., 2009, Vol. 23, pp. 869-883.

25. Chen X.F., Yang Z.B., Zhang X.W., He Z.J. Modeling of wave propagation in onedimension structures using B-spline wavelet on interval finite element. // Finite Elem. Anal. Des., 2012, Vol. 51, pp. 1-9.

26. Han J.G., Ren W.X., Huang Y. A multivariable wavelet-based finite element method and its application to thick plates. // Finite Elem. Anal. Des., 2005, Vol. 41, pp. 821-833.

27. Zhang X.W., Chen X.F., He Z.J. The analysis of shallow shells based on multivariable wavelet finite element method. // Acta Mech. Solida Sin., 2011, Vol. 24, pp. 450-460.

28. Han J.G., Ren W.X., Huang Y. A waveletbased stochastic finite element method of thin plate bending. // Appl. Math. Model., 2007, Vol. 31, pp. 181-193.

29. Pian T.H.H., Chen D.P. Alternative ways for formulation of hybrid stress elements. // Int. J. Numer. Methods Eng., 1982, Vol. 18, pp. 1679-1684.

30. Pian T.H.H., Sumihara K. Rational approach for assumed stress finite elements. // Int. J. 
Localization of Solution of the Problem of Two-Dimensional Theory of Elasticity with the Use of B-Spline Discrete-

Continual Finite Element Method

Numer. Methods Eng., 1984, Vol. 20, pp. $1685-1695$.

31. Mau S.T., Tong P., Pian T.H.H. Finite element solutions for laminated thick plates. // J. Compos. Mater., 1972, Vol. 6, pp. 304-311.

32. Akimov P.A. Correct Discrete-Continual Finite Element Method of Structural Analysis Based on Precise Analytical Solutions of Resulting Multipoint Boundary Problems for Systems of Ordinary Differential Equations. // Applied Mechanics and Materials, 2012, Vols. 204-208, pp. 4502-4505.

33. Akimov P.A., Belostotskiy A.M., Mozgaleva M.L., Mojtaba Aslami, Negrozov O.A. Correct Multilevel Discrete-Continual Finite Element Method of Structural Analysis. // Advanced Materials Research, 2014, Vol. 1040, pp. 664-669.

34. Zolotov A.B., Akimov P.A. Semianalytical Finite Element Method for Two-dimensional and Three-dimensional Problems of Structural Analysis. // Proceedings of the International Symposium LSCE 2002 organized by Polish Chapter of IASS, Warsaw, Poland, 2002, pp. 431-440.

35. Akimov P.A., Sidorov V.N. Correct Method of Analytical Solution of Multipoint Boundary Problems of Structural Analysis for Systems of Ordinary Differential Equations with Piecewise Constant Coefficients. // Advanced Materials Research, 2011, Vols. 250-253, pp. 3652-3655.

36. Akimov P.A., Mozgaleva M.L. Correct Wavelet-based Multilevel Discrete-Continual Methods for Local Solution of Boundary Problems of Structural Analysis. // Applied Mechanics and Materials, 2013, Vols. 353356, pp. 3224-3227.

37. Akimov P.A., Mozgaleva M.L. Waveletbased Multilevel Discrete-Continual Finite Element Method for Local Deep Beam Analysis. // Applied Mechanics and Materials, 2013, Vols. 405-408, pp. 3165-3168.

38. Akimov P.A., Mozgaleva M.L. Waveletbased Multilevel Discrete-Continual Finite Element Method for Local Plate Analysis. //
Applied Mechanics and Materials, 2013, Vols. 351-352, pp. 13-16.

39. Akimov P.A., Mozgaleva M.L., Mojtaba Aslami, Negrozov O.A. Modified Waveletbased Multilevel Discrete-Continual Finite Element Method for Local Structural Analysis. Part 1: Continual and Discrete-Continual Formulations of the Problems. // Applied Mechanics and Materials, 2014, Vols. 670671, pp.720-723.

40. Akimov P.A., Mozgaleva M.L., Mojtaba Aslami, Negrozov O.A. Modified Waveletbased Multilevel Discrete-Continual Finite Element Method for Local Structural Analysis. Part 2: Reduced Formulations of the Problems in Haar Basis. // Applied Mechanics and Materials, 2014, Vols. 670671, pp. 724-727.

41. Akimov P.A., Mozgaleva M.L., Mojtaba Aslami, Negrozov O.A. Wavelet-Based Discrete-Continual Finite Element Method of Local Structural Analysis for Two-Dimensional Problems. // Procedia Engineering, 2014, Vol. 91, pp. 8-13.

42. Mozgaleva M.L., Akimov P.A. About Verification of Wavelet-Based DiscreteContinual Finite Element Method for ThreeDimensional Problems of Structural Analysis. Part 1: Structures with Constant Physical and Geometrical Parameters Along Basic Direction. // Applied Mechanics and Materials, 2015, Vol. 709, pp. 105-108.

43. Mozgaleva M.L., Akimov P.A. About Verification of Wavelet-Based DiscreteContinual Finite Element Method for ThreeDimensional Problems of Structural Analysis. Part 2: Structures with Piecewise Constant Physical and Geometrical Parameters Along Basic Direction. // Applied Mechanics and Materials, 2015, Vol. 709, pp. 109-112.

44. Akimov P.A., Mozgaleva M.L. Method of Extended Domain and General Principles of Mesh Approximation for Boundary Problems of Structural Analysis. // Applied Mechanics and Materials, 2014, Vols. 580-583, pp. 2898-2902. 


\section{СПИСОК ЛИТЕРАТУРЫ}

1. Akimov P.A., Mozgaleva M.L., Kaytukov T.B. Numerical solution of the problem of isotropic plate analysis with the use of B-spline discrete-continual finite element method. // // International Journal for Computational Civil and Structural Engineering, 2020, Vol. 16, Issue 4, pp. 14-28.

2. Akimov P.A., Mozgaleva M.L., Kaytukov T.B. Numerical solution of the problem of beam analysis with the use of B-spline finite element method. // International Journal for Computational Civil and Structural Engineering, 2020, Vol. 16, Issue 3, pp. 12-22.

3. Li B., Chen X. Wavelet-based numerical analysis: A review and classification. // Finite Elements in Analysis and Design, 2014, Vol. 81, pp. 14-31.

4. Daubechies I. Orthonormal bases of compactly supported wavelets. // Commun. Pure Appl. Math., 1988, Vol. 41, pp. 909-996.

5. Li B., Cao H.R., He Z.J. The construction of one-dimensional Daubechies wavelet-based finite elements for structural response analysis. // J. Vibroeng, 2011, vol. 13, pp. 729-738.

6. Ma J.R., Xue J.J. A study of the construction and application of a Daubechies wave-letbased beam element. // Finite Elements in Analysis and Design, 2003, Vol. 39, pp. 965-975.

7. Mozgaleva M.L., Akimov P.A., Kaytukov T.B. About wavelet-based computational beam analysis with the use of Daubechies scaling functions. // International Journal for Computational Civil and Structural Engineering, 2019, Vol. 15, Issue 2, pp. 95-110.

8. Mozgaleva M.L., Akimov P.A., Kaytukov T.B. Wavelet-based discrete-continual finite element plate analysis with the use of Daubechies scaling functions. // International Journal for Computational Civil and Structural Engineering, 2019, Vol. 15, Issue 3, pp. 96-108.

9. Antes H. Bicubic fundamental splines in plate bending. // Int. J. Numer. Methods Eng., 1974, Vol. 8, pp. 503-511.
10. Han J.G., Ren W.X., Huang Y. A spline wavelet finite-element method in structural mechanics. // Int. J. Numer. Methods Eng., 2006, Vol. 66, pp. 166-190.

11. Han J.G., Ren W.X., Huang Y. A spline wavelet finite element formulation of thin plate bending. // Eng. Comput., 2009, Vol. 25, pp. 319-326.

12. Xiang J.W., Chen X.F., He Z.J., Zhang Y.H. A new wavelet-based thin plate element using B-spline wavelet on the interval. // Comput. Math., 2008, Vol. 41, pp. 243-255.

13. Chen X.F., Xiang J.W., Li B., He Z.J. A study of multiscale wavelet-based elements for adaptive finite element analysis. // Adv. Eng. Softw., 2010, Vol. 41, pp. 196-205.

14. Zhong Y.T., Xiang J.W. Construction of wavelet-based elements for static and stability analysis of elastic problems. // Acta Mech. Solida Sin., 2011, Vol. 24, pp. 355-364.

15. Yang Z.B., Chen X.F., Li B., He Z.J., Miao H.H. Vibration analysis of curved shell using b-spline wavelet on the interval (BSWI). // Finite Elements Method and General Shell Theory, CMES85, 2012, pp. 129-155.

16. Yang Z.B., Chen X.F., Zhang X.W., He Z.J. Free vibration and buckling analysis of plates using B-spline wavelet on the interval Mindlin element. // Appl. Math. Model., 2013, Vol. 37, pp. 3449-3466.

17. Xiang J.W., Chen X.F., Li B., He Y.M., He Z.J. Identification of a crack in a beam based on the finite element method of a B-spline wavelet on the interval. // J. Sound Vibr., 2006, Vol. 296, pp. 1046-1052.

18. Xiang J.W., Chen X.F., Mo Q.M., He Z.H. Identification of crack in a rotor system based on wavelet finite element method. // Finite Elem. Anal. Des., 2007, Vol. 43, pp. 1068-1081.

19. Xiang J.W., Liang M. A two-step approach to multi-damage detection for plate structures. // Eng. Fract. Mech., 2012, Vol. 91, pp. 73-86.

20. Xiang J.W., Matsumoto T., Long J.Q., Ma G. Identification of damage locations based on operating deflection shape. // Nondestruct. Test. Eval., 2012, Vol. 1, pp. 1-15. 
21. Xiang J.W., Liang M. Wavelet-based detection of beam cracks using modal shape and frequency measurements. // Comput.Aided Civil Infrastruct. Eng., 2012, Vol. 27, pp. 439-454.

22. Xiang J.W., Matsumoto T., Wang Y.W., Jiang Z.S. Detect damages in conical shells using curvature mode shape and wavelet finite element method. // Int. J. Mech. Sci., 2013, Vol. 66, pp. 83-93.

23. Dong H.B., Chen X.F., Li B., Qi K.Y., He Z.J. Rotor crack detection based on highprecision modal parameter identification method and wavelet finite element model. // Mech. Syst. Signal Process., 2009, Vol. 23, pp. 869-883.

24. Chen X.F., Yang Z.B., Zhang X.W., He Z.J. Modeling of wave propagation in onedimension structures using B-spline wavelet on interval finite element. // Finite Elem. Anal. Des., 2012, Vol. 51, pp. 1-9.

25. Han J.G., Ren W.X., Huang Y.Amultivariable wavelet-based finite element method and its application to thick plates. // Finite Elem. Anal. Des., 2005, Vol. 41, pp. 821-833.

26. Zhang X.W., Chen X.F., He Z.J. The analysis of shallow shells based on multivariable wavelet finite element method. // Acta Mech. Solida Sin., 2011, Vol. 24, pp. 450-460.

27. Han J.G., Ren W.X., Huang Y. A waveletbased stochastic finite element method of thin plate bending. // Appl. Math. Model., 2007, Vol. 31, pp. 181-193.

28. Pian T.H.H., Chen D.P. Alternative ways for formulation of hybrid stress elements. // Int. J. Numer. Methods Eng., 1982, Vol. 18, pp. 1679-1684.

29. Pian T.H.H., Sumihara K. Rational approach for assumed stress finite elements. // Int. J. Numer. Methods Eng., 1984, Vol. 20, pp. 1685-1695.

30. Mau S.T., Tong P., Pian T.H.H. Finite element solutions for laminated thick plates. // J. Compos. Mater., 1972, Vol. 6, pp. 304-311.

31. Akimov P.A. Correct Discrete-Continual Finite Element Method of Structural Analysis
Based on Precise Analytical Solutions of Resulting Multipoint Boundary Problems for Systems of Ordinary Differential Equations. // Applied Mechanics and Materials, 2012, Vols. 204-208, pp. 4502-4505.

32. Akimov P.A., Belostotskiy A.M., Mozgaleva M.L., Mojtaba Aslami, Negrozov O.A. Correct Multilevel Discrete-Continual Finite Element Method of Structural Analysis. // Advanced Materials Research, 2014, Vol. 1040, pp. 664-669.

33. Zolotov A.B., Akimov P.A. Semianalytical Finite Element Method for Two-dimensional and Three-dimensional Problems of Structural Analysis. // Proceedings of the International Symposium LSCE 2002 organized by Polish Chapter of IASS, Warsaw, Poland, 2002, pp. 431-440.

34. Akimov P.A., Sidorov V.N. Correct Method of Analytical Solution of Multipoint Boundary Problems of Structural Analysis for Systems of Ordinary Differential Equations with Piecewise Constant Coefficients. // Advanced Materials Research, 2011, Vols. 250-253, pp. 3652-3655.

35. Akimov P.A., Mozgaleva M.L. Correct Wavelet-based Multilevel Discrete-Continual Methods for Local Solution of Boundary Problems of Structural Analysis. // Applied Mechanics and Materials, 2013, Vols. 353356, pp. 3224-3227.

36. Akimov P.A., Mozgaleva M.L. Waveletbased Multilevel Discrete-Continual Finite Element Method for Local Deep Beam Analysis. // Applied Mechanics and Materials, 2013, Vols. 405-408, pp. 3165-3168.

37. Akimov P.A., Mozgaleva M.L. Waveletbased Multilevel Discrete-Continual Finite Element Method for Local Plate Analysis. // Applied Mechanics and Materials, 2013, Vols. 351-352, pp. 13-16.

38. Akimov P.A., Mozgaleva M.L., Mojtaba Aslami, Negrozov O.A. Modified Waveletbased Multilevel Discrete-Continual Finite Element Method for Local Structural Analysis. Part 1: Continual and Discrete-Continual 
Formulations of the Problems. // Applied Mechanics and Materials, 2014, Vols. 670671, pp.720-723.

39. Akimov P.A., Mozgaleva M.L., Mojtaba Aslami, Negrozov O.A. Modified Waveletbased Multilevel Discrete-Continual Finite Element Method for Local Structural Analysis. Part 2: Reduced Formulations of the Problems in Haar Basis. // Applied Mechanics and Materials, 2014, Vols. 670671, pp. 724-727.

40. Akimov P.A., Mozgaleva M.L., Mojtaba Aslami, Negrozov O.A. Wavelet-Based Discrete-Continual Finite Element Method of Local Structural Analysis for Two-Dimensional Problems. // Procedia Engineering, 2014, Vol. 91, pp. 8-13.

41. Mozgaleva M.L., Akimov P.A. About Verification of Wavelet-Based DiscreteContinual Finite Element Method for Three-
Dimensional Problems of Structural Analysis. Part 1: Structures with Constant Physical and Geometrical Parameters Along Basic Direction. // Applied Mechanics and Materials, 2015, Vol. 709, pp. 105-108.

42. Mozgaleva M.L., Akimov P.A. About Verification of Wavelet-Based DiscreteContinual Finite Element Method for ThreeDimensional Problems of Structural Analysis. Part 2: Structures with Piecewise Constant Physical and Geometrical Parameters Along Basic Direction. // Applied Mechanics and Materials, 2015, Vol. 709, pp. 109-112.

43. Akimov P.A., Mozgaleva M.L. Method of Extended Domain and General Principles of Mesh Approximation for Boundary Problems of Structural Analysis. // Applied Mechanics and Materials, 2014, Vols. 580-583, pp. 2898-2902.
Pavel A. Akimov, Full Member of the Russian Academy of Architecture and Construction Sciences, Professor, Dr.Sc.; Acting Rector of National Research Moscow State University of Civil Engineering; Professor of Department of Architecture and Construction, Peoples' Friendship University of Russia; Professor of Department of Structural Mechanics, Tomsk State University of Architecture and Building; Acting Vice-President of the Russian Academy of Architecture and Construction Sciences; 26, Yaroslavskoe Shosse, Moscow, 129337, Russia; phone: +7(495) 651-8185; Fax: +7(499) 183-44-38; E-mail:AkimovPA@mgsu.ru, rector@mgsu.ru, pavel.akimov@gmail.com.

Marina L. Mozgaleva, Senior Scientist Researcher, Dr.Sc.; Professor of Department of Applied Mathematics, National Research Moscow State University of Civil Engineering; 26, Yaroslavskoe Shosse, Moscow, 129337, Russia; phone/fax +7(499) 183-59-94; Fax: +7(499) 183-44-38; Email: marina. mozgaleva@gmail.com.

Taymuraz B. Kaytukov, Advisor of the Russian Academy of Architecture and Construction Sciences, Associate Professor, Ph.D.; Vice-Rector, Associate Professor Department of Applied Mathematics, National Research Moscow State University of Civil Engineering; 26, Yaroslavskoe Shosse, Moscow, 129337, Russia; phone: +7(499) 929-52-29; fax: +7(499) 183-59-94; Email: KaytukovTB@mgsu.ru.
Акимов Павел Алексеевич, академик РААСН, профессор, доктор технических наук; ректор Национального исследовательского Московского государственного строительного университета; профессор Департамента архитектуры и строительства Российского университета дружбы народов; профессор кафедры строительной механики Томского государственного архитектурно-строительного университета; исполняющий обязанности вице-президента Российской академии архитектуры и строительных наук; 129337, Россия, г. Москва, Ярославское шоссе, дом 26; телефон: +7(495) 651-81-85; факс: +7(499) 183-44-38; Email: AkimovPA@ mgsu.ru, rector@mgsu.ru, pavel.akimov@gmail.com.

Мозгалева Марина Леонидовна, старший научный сотрудник, доктор технических наук; профессор кафедры прикладной математики Национального исследовательского Московского государственного строительного университета; 129337, Россия, г. Москва, Ярославское шоссе, дом 26; телефон/факс: +7(499) 183-59-94; Email: marina.mozgaleva@gmail.com.

Кайтуков Таймураз Батразович, советник РААСН, доцент, кандидат технических наук; проректор, профессор кафедры прикладной математики Национального исследовательского Московского государственного строительного университета; 129337, Россия, г. Москва, Ярославское шоссе, дом 26; телефон: +7(499) 929-52-29; факс: +7(499) 183-44-38; Email: KaytukovTB@mgsu.ru. 